\title{
Phosphino-Triazole Ligands for Palladium-Catalyzed Cross-Coupling
}

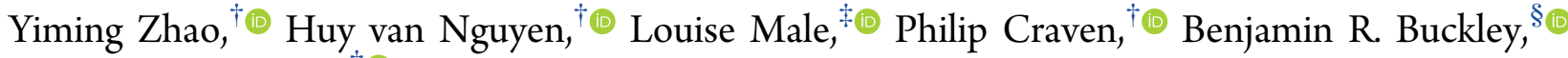
and John S. Fossey* ${ }^{* \dagger}$

${ }^{\dagger}$ School of Chemistry and ${ }^{\ddagger}$ X-ray Crystallography Facility, School of Chemistry, University of Birmingham, Edgbaston, Birmingham, West Midlands B15 2TT, United Kingdom

${ }^{\S}$ Department of Chemistry, Loughborough University, Loughborough, Leicestershire LE11 3TU, United Kingdom

\section{Supporting Information}

ABSTRACT: Twelve 1,5-disubtituted and fourteen 5-substituted 1,2,3-triazole derivatives bearing diaryl or dialkyl phosphines at the 5-position were synthesized and used as ligands for palladium-catalyzed Suzuki-Miyaura cross-coupling reactions. Bulky substrates were tested, and lead-like product formation was demonstrated. The online tool SambVca2.0 was used to assess steric parameters of ligands and preliminary buried volume determination using XRDobtained data in a small number of cases proved to be informative. Two modeling approaches were compared for the determination of the buried volume of ligands where XRD data was not available. An approach with imposed steric restrictions was found to be superior in leading to buried volume determinations that closely correlate with observed reaction conversions. The online tool LLAMA was used to determine lead-likeness of potential Suzuki-Miyaura cross-coupling products, from which 10 of the most lead-like were successfully synthesized. Thus, confirming these readily accessible triazole-containing phosphines as highly suitable ligands for reaction screening and optimization in drug discovery campaigns.

\section{INTRODUCTION}

Click chemistry, as defined by Sharpless and co-workers, ${ }^{1-4}$ has transformed the face, and accessibility to the nonspecialist of molecular linking strategies. Among click approaches, the highly regioselective copper-catalyzed, Huisgen cycloaddition reaction to form 1,4-triazoles (Figure 1, 1) by Meldal and

$$
\mathrm{R}^{1}=+\mathrm{N}_{3}-\mathrm{R}^{2} \stackrel{\text { Cu cat. }}{\longrightarrow} \underbrace{\mathrm{R}-\mathrm{R}^{2}}_{\mathrm{N}=\mathrm{N}^{\prime}}
$$

Figure 1. Upper: copper-catalyzed azide-alkyne cycloaddition (CuAAC). Lower: examples of 1,2,3-triazole-containing structures.

Sharpless, ${ }^{4,5}$ has grown in popularity and has been employed in increasingly varied applications over the intervening years. ${ }^{6}$ The copper-catalyzed azide-alkyne cycloaddition (CuAAC) is ubiquitous, , $^{5-9}$ and often referred to as "the click reaction", and the product 1,2,3-triazoles, bearing 1,4-substition patterns, with reliable fidelity and yields being synonymous with click chemistry (Figure 1, 1). ${ }^{8,10-15}$ However, the resulting triazoles are not always employed as innocent bystander linkage motifs. Triazoles of this type have been used as analogues of peptide linkages (Figure 1, 2), ${ }^{16}$ such peptidomimetics are physiologically stable, and their modular synthesis allows access to a broad range of biologically relevant applications. ${ }^{17,18}$ The utility of further synthetic transformations has been probed, particularly in the derivatization at the 5-position (Figure 1, 3). ${ }^{19,20}$

Copper-catalyzed triazole formation has been exploited in a wide range of scenarios ${ }^{21}$ and has been the subject of various mechanistic studies, ${ }^{22-26}$ leading to the proposal of a binuclear transition state involving two copper atoms. 1,2,3-Triazole derivatives have been employed as nitrogen-coordinating ligands, ${ }^{27}$ e.g. in $\mathrm{N}, \mathrm{N}^{\prime}-{ }^{28-34} \mathrm{~N}, \mathrm{~S}-{ }^{29}{ }^{29} \mathrm{~N}, \mathrm{Se}^{2}{ }^{29}$ and cyclometalated $^{35-37}$ bidentate coordination complexes. Furthermore, tris-triazoles, such as TBTA (Figure 2, 4) and its analogues, have been used as ligands for reactions including the CuAAC by both Fokin ${ }^{38}$ and $\mathrm{Zhu}^{39}$ and their co-workers, demonstrating exceptional ligand-mediated reaction acceleration.

Alkylation of a 1,2,3-triazole derivative, to furnish a 1,3,4trisubstituted triazolium salt, is the first step in the synthesis of a newer class of $\mathrm{N}$-heterocyclic carbene (NHC) reported in

Received: July 30, 2018

Published: October 17, 2018 


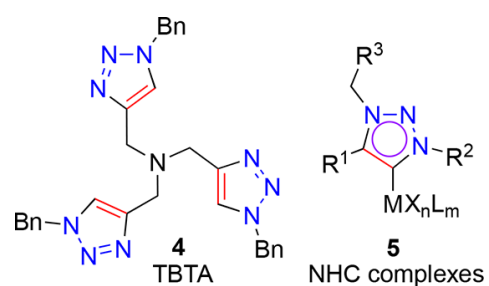

Figure 2. Examples of triazole-containing or -derived ligands.

2008 by Albrecht and co-workers ${ }^{40}$ and later extended by Lee and Crowley ${ }^{41-43}$ as ligands for gold and by Grubbs and Bertrand as ligands for ruthenium-mediated catalysis (Figure 2, 5). ${ }^{44}$ The ready access to a range of ligand scaffolds through the $\mathrm{CuAAC}$ has led the triazole-NHC platform to continue to gain in popularity. ${ }^{45-49}$ These reports demonstrate the 1,2,3triazole unit is a legitimate candidate for further exploitation as a key component in ligand design and catalyst development. ${ }^{50}$

The co-authors of this report have investigated triazoles as chemosensors $^{51-53}$ and as products of asymmetric synthesis. ${ }^{54-58}$ Furthermore, an interest in boronic acid derivatives as chemosensors, ${ }^{59-64}$ including the use of cross-coupling reactions as a means of sensing, ${ }^{63}$ means the co-authors of this report are familiar with boronic acids and esters. ${ }^{66}$ As such, a desire to bring together these streams of research under one umbrella has led to the research reported in this manuscript. Herein, the development of bulky and highly active triazolecontaining phosphine ligands for palladium-catalyzed SuzukiMiyaura cross-coupling reactions is explored (Scheme 1).

Scheme 1. Outline of a General Palladium-Catalyzed Suzuki-Miyaura Formation of Biaryl 6

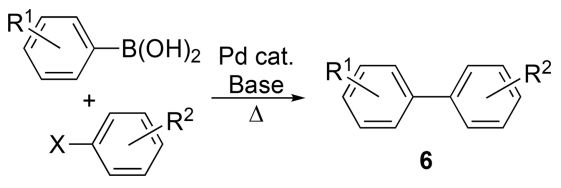

Palladium-catalyzed cross-coupling reactions are well-studied and offer ready access to an extensive range of (bi)aryl motifs (Scheme 1, 6)..$^{67-76}$ The suite of palladium-catalyzed cross-coupling chemistry available for the construction of biologically relevant products has transformed the field of medicinal chemistry, yet cross-coupling between sterically hindered and lead-like building blocks, particularly with aryl chlorides, remains challenging.

Developments in palladium-catalyzed chemistry have been heavily influenced by ligand design and optimization. Among the superior ligands for palladium-catalyzed cross-coupling reactions are bulky alkyl phosphines, ${ }^{78-82}$ and bulky orthosubstituted aryl-alkyl phosphines, ${ }^{83,84}$ such as S-Phos (Figure 3, 7) ${ }^{85}$ and X-Phos (Figure 3, 8). ${ }^{86}$ Metallocyclic precatalysts have been developed which delivered greater stability, more facile manipulation and enhanced reaction outcomes. ${ }^{87-89}$

Phosphines appended to one or more five-membered, all $\mathrm{sp}^{2}$ rings have been shown to offer advantages in some cases. The pyrrole-appended phosphines of Beller and co-workers (Figure $3,9)^{90,91}$ have proven to be useful ligands in cross-coupling catalysis that furnishes drug-like products. Furthermore, the bulky, electron-rich, ferrocene-appended phosphines of Richards (Figure 3, 10), ${ }^{92}$ Johannsen (Figure 3, 11), ${ }^{93} \mathrm{Fu}$ (Figure 3, 12), ${ }^{94}$ and their respective co-workers provide

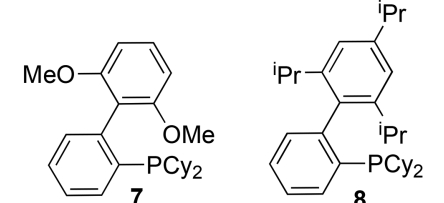

Buchwald S-Phos Buchwald X-Phos

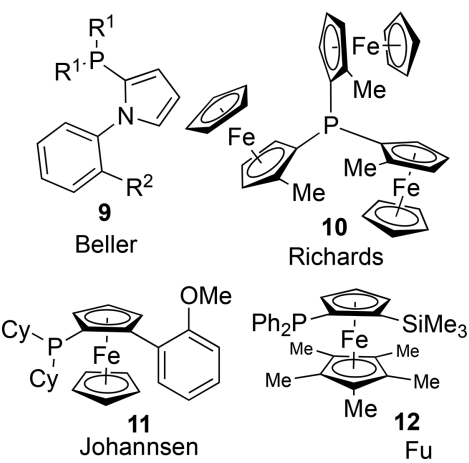

Figure 3. Representative examples of bulky phosphine ligands including: upper: S-Phos (7) and X-Phos (8); middle left: heteroaromatic phosphine derivative (9); middle right and lower row: Phosphino-ferrocene derivatives displaying planar chirality.

access to highly active palladium-ligand conjugates for crosscoupling some of the least active substrates.

1,4-Disubstituted-1,2,3-triazoles have been used in the assembly of phosphorus-containing species that have the potential to be employed as ligands. Ready access to libraries of products using CuAAC reactions has permitted the development of phosphines connected to triazoles with $\mathrm{sp}^{3}$ linkages to the 1-N and 4-C positions. Examples include those reported by Dubrovnia, Börner and co-workers (Figure 4, 13), ${ }^{95}$ Gandelman and co-workers who prepared $\mathrm{PCP}^{\prime}$ pincer complexes from bis-phosphinotriazoles (Figure 4, 14), ${ }^{96}$ and Kann and

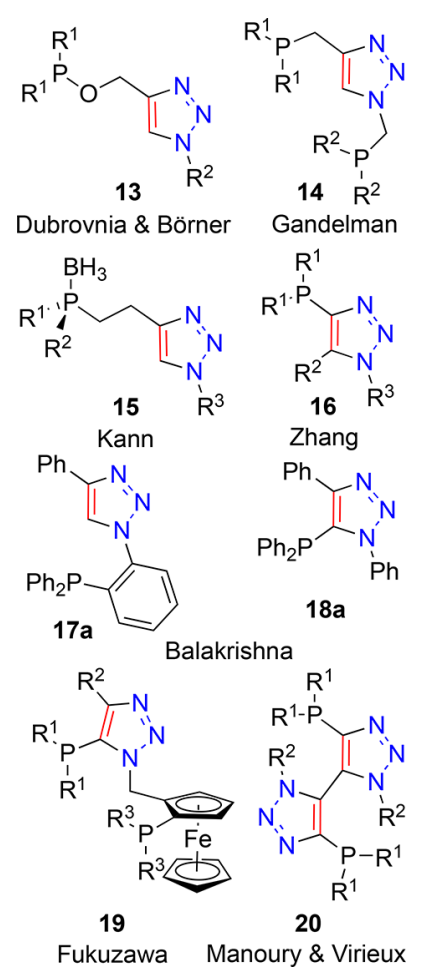

Figure 4. Phosphorus-containing 1,2,3-triazole derivatives. 
co-workers who used a borane-protected $P$-chiral azide to deliver protected $P$-chiral triazole-containing phosphines (Figure 4, 15). ${ }^{97}$ Since proximal stoichiometric phosphine can arrest the CuAAC through coordinative saturation of substoichiometic copper catalyst or unwanted Staudinger-type reactions between phosphine and azide derivative, alternative approaches are required to deliver $P$-appended triazoles. Zhang and co-workers reported on the use of alkynyl Grignard reagents for the synthesis of a phosphine series where phosphorus is attached directly to a 1,2,3-triazole ring at the 4-position (Figure 4, 16). ${ }^{98-101}$ As part of an impressive, rigorous, and detailed study, Balakrishna and co-workers prepared not only expected aryl phosphine triazole derivative 17a (Figure 4) through lithium-halogen exchange and subsequent reaction with diphenylphosphorus chloride on the corresponding bromide under kinetic control but also thermodynamically favored 5-phosphino triazole 18a (Figure 4) from the same aryl bromide starting material under different conditions. $^{102}$ Fukuzawa and co-workers also employed deprotonation of the 5-position of a 1,2,3-triazole to facilitate installation of 5-phosphino functionality in their ferroncenyl bisphosphino ligand synthesis (Figure 4, 19). ${ }^{103}$ Glover et al. ${ }^{104}$ and Austeri et al. ${ }^{105}$ also utilized deprotonation of the triazole 5-position to create planar chiral cyclophanecontaining analogues of 18a. In order to successfully synthesize a bis-5-phosphino-triazole bidentate ligand, Manoury, Virieux, and co-workers employed an ethynyl phosphine oxide in their homocoupled dimer synthesis, which after treatment with trichlorosilane resulted in 20 (Figure 4). ${ }^{106}$ A structurally related 5-18 hybrid system was also recently reported by Cao et al., who showed the formation of bimetallic complexes of NHC-phosphine mixed systems. ${ }^{107}$

Bulky, or sterically hindered, phosphorus-containing ligands have also found application outside of the palladium catalysis arena; steric parameters appear to be important in a variety of gold-mediated transformations. ${ }^{108-110}$ Steric and electronic parameters of phosphine ligands have been a subject of study for more than 40 years. ${ }^{11}$ More recent contributions have built upon Tolman's concept of cone angle as a descriptor of the steric bulk a ligand imparts about a metal, resulting in a parameter known as buried volume $\left(\% V_{\text {Bur }}\right)$ coming to the fore. ${ }^{112}$ Cavallo and co-workers have developed a free webbased tool for the calculation of $\% V_{\text {Bur }}$, named SambVca. ${ }^{13}$ This parametrization of ligands, using both spectroscopic measurements and calculated properties (using principle component analysis for example), has facilitated exceptional ligand design and optimization across a range of catalyzed reactions. $^{114-116}$

While a range of ligand-based solutions for cross-coupling reactions exist, a platform to rapidly deliver alternatives and explore chemical space around novel catalyst constructs, such as through $\mathrm{CuAAC}$, offers approaches and complementary tools to the field. Furthermore, cross-coupling catalysis manifolds that provide access to increasingly three-dimensional products, ${ }^{117}$ and those directly delivering products with leador drug-like properties ${ }^{118}$ without need of protecting group removal or further derivatization are desired and less-well explored. $^{77}$

\section{RESULTS AND DISCUSSION}

In order to overcome the general incompatibility of phosphines with the CuAAC reaction, an approach other than direct phosphine incorporation is required. While
Scheme 2. Reaction of 1,2,3-Triazoles $21 \mathrm{a}-\mathrm{d}$ with $n$ Butyllithium Followed by Treatment with Diphenyl Phosphorous Chloride Led Primarily to Formation of Phosphino-Triazole 18a
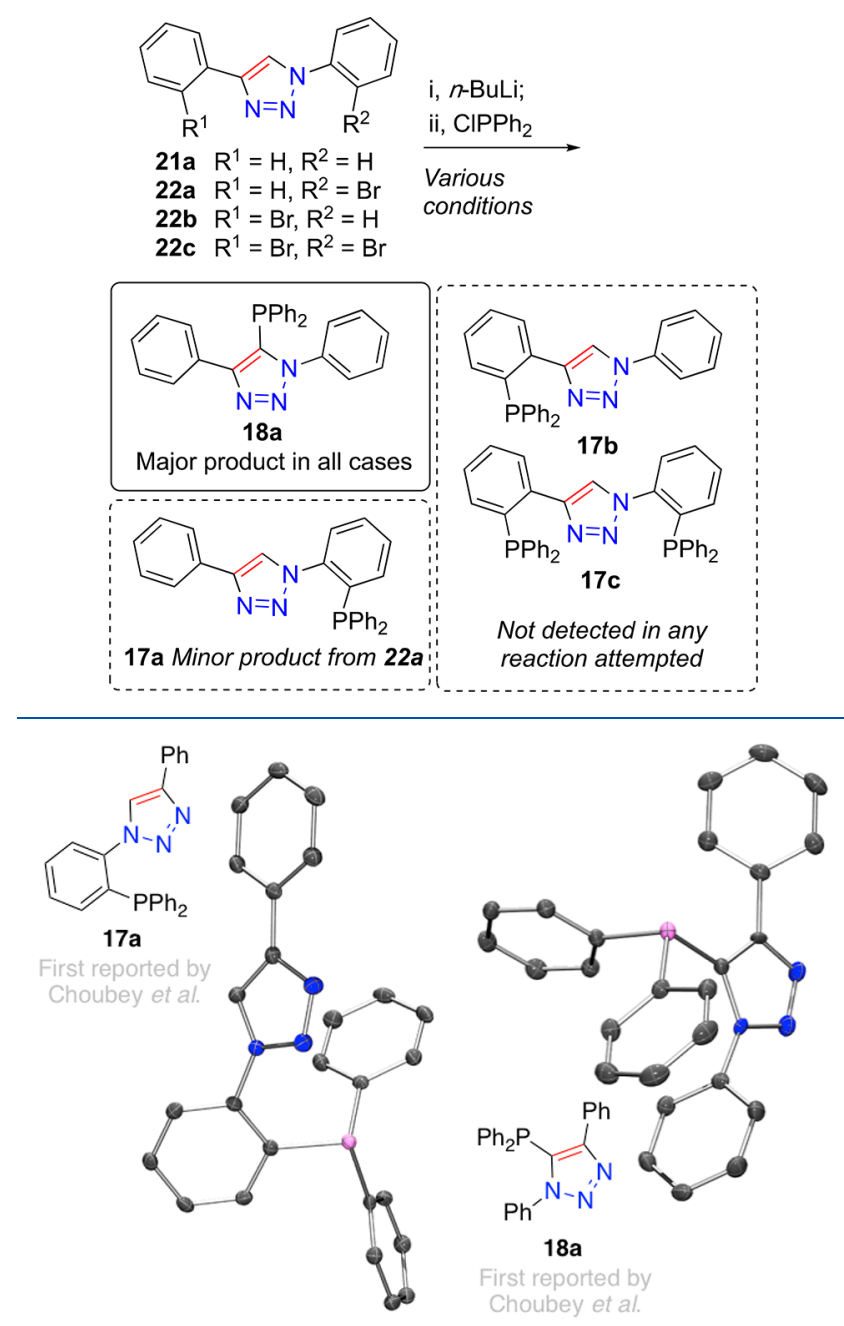

Figure 5. Representation of the crystal structures of isomeric 17a (left) and 18a (right), ellipsoids drawn at the $50 \%$ probability level (Ortep3 for Windows and PovRay). For 17a the structure contains two crystallographically independent molecules with only one shown for clarity. For 18a the phenyl-appended 1-nitrogen and 4-carbon atoms of the triazole unit are disordered such that the triazole ring occupies two opposing orientations, related by a $180^{\circ}$ rotation of the triazole ring about the phosphorus-triazole bond. The refined percentage occupancy ratio of the two positions are 59.7 (15) and 40.3 (15); one arbitrary molecule depicted and hydrogen atoms removed for clarity.

protection of the phosphine (as a phosphine oxide for example $)^{106}$ is possible, the initial approach chosen in this program was to probe the potential for triazole-mediated directed ortho-lithiation or halogen-lithium exchange, and subsequent reaction with diphenyl phosphorus chloride, as possible routes to phosphino-triazoles $17 \mathbf{a}-\mathbf{c}$. Accordingly, 1,4-diphenyl-1,2,3-triazole (21a) was selected as a model substrate to test if ortho-lithiation could deliver the required intermediate to give the desired product $17 \mathbf{a}$. As such, 21a was reacted at $-78{ }^{\circ} \mathrm{C}$ with $n$-butyllithium, followed by addition of the phosphorus chloride reagent, before being allowed to warm to room temperature, and being allowed to stir for a period of 
Scheme 3. Previously Reported Kinetic (Upper) and Thermodynamic (Lower) Lithiation and Subsequent Phosphorous Addition to Brominated Triazole 22a Affording P-Aryl and P-Triazole Derivatives, Respectively ${ }^{a}$

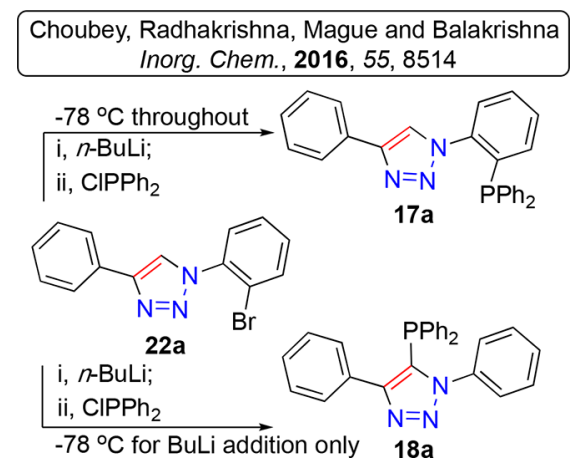

${ }^{a} \operatorname{Ref} 102$.

Scheme 4. CuAAC Reaction to Form 1,4-Disubstituted 1,2,3-Triazole Derivatives 21a-i

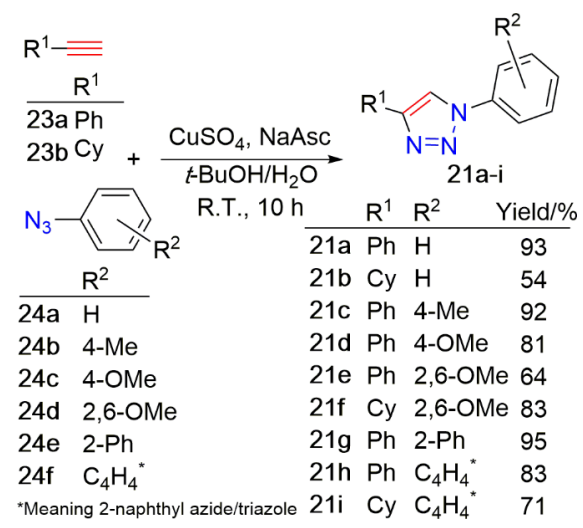

Scheme 5. Deprotonation and Subsequent Reaction with Phosphorous Chloride Reagent Protocol for Delivery of $18 \mathrm{a}-1$

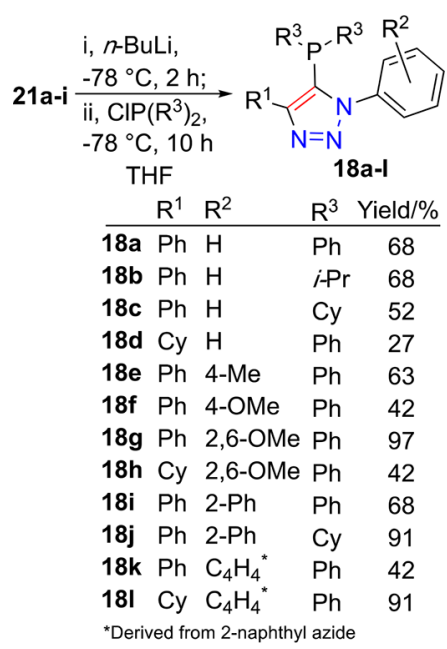

time. Over numerous attempts only the unanticipated product 18a was isolated from such reactions, Scheme 2. This indicates that deprotonation of the 5-position of the triazole is facile, resulting in formation of the observed major product. In order to mitigate against the formation of unanticipated triazolephosphine 18a, brominated triazole derivatives $22 \mathrm{a}-\mathrm{c}$ were
Table 1. Ligand Screening: 1,4-Disubstituted 1,2,3-TriazoleContaining Phosphine Ligand Mediate, PalladiumCatalyzed, Formation of $27^{a}$
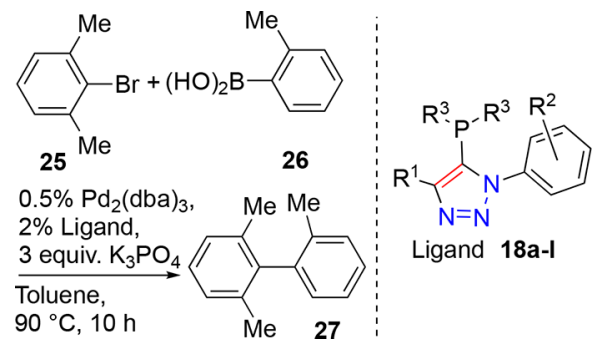

\begin{tabular}{clllcc} 
entry & $\mathrm{R}^{1}$ & \multicolumn{1}{c}{$\mathrm{R}^{2}$} & $\mathrm{R}^{3}$ & ligand & conversion [\%] \\
$\mathbf{1}$ & & & & $\mathbf{1 7 a}$ & 29 \\
$\mathbf{2}$ & $\mathrm{Ph}$ & $\mathrm{H}$ & $\mathrm{Ph}$ & $\mathbf{1 8 a}$ & 47 \\
$\mathbf{3}$ & $\mathrm{Ph}$ & $\mathrm{H}$ & $i-\mathrm{Pr}$ & $\mathbf{1 8 b}$ & 62 \\
$\mathbf{4}$ & $\mathrm{Ph}$ & $\mathrm{H}$ & $\mathrm{Cy}$ & $\mathbf{1 8 c}$ & 75 \\
$\mathbf{5}$ & $\mathrm{Cy}$ & $\mathrm{H}$ & $\mathrm{Ph}$ & $\mathbf{1 8 d}$ & 86 \\
$\mathbf{6}$ & $\mathrm{Ph}$ & 4-Me & $\mathrm{Ph}$ & $\mathbf{1 8 e}$ & 69 \\
$\mathbf{7}$ & $\mathrm{Ph}$ & 4-OMe & $\mathrm{Ph}$ & $\mathbf{1 8 f}$ & 83 \\
$\mathbf{8}$ & $\mathrm{Ph}$ & 2,6-OMe & $\mathrm{Ph}$ & $\mathbf{1 8 g}$ & 92 \\
$\mathbf{9}$ & $\mathrm{Cy}$ & 2,6-OMe & $\mathrm{Ph}$ & $\mathbf{1 8 h}$ & 90 \\
$\mathbf{1 0}$ & $\mathrm{Ph}$ & 2-Ph & $\mathrm{Ph}$ & $\mathbf{1 8 i}$ & 84 \\
$\mathbf{1 1}$ & $\mathrm{Ph}$ & 2-Ph & $\mathrm{Cy}$ & $\mathbf{1 8 j}$ & 92 \\
$\mathbf{1 2}$ & $\mathrm{Ph}$ & 2-Napth & $\mathrm{Ph}$ & $\mathbf{1 8 k}$ & 91 \\
$\mathbf{1 3}$ & $\mathrm{Cy}$ & 2-Napth & \\
& & $\mathrm{Ph}$ & $\mathbf{1 8 1}$ & 99
\end{tabular}

${ }^{a}$ Reaction conditions: 2 -bromo- $m$-xylene $(0.4 \mathrm{mmol}), o$-tolylboronic acid $(0.6 \mathrm{mmol})$, potassium phosphate $(1.2 \mathrm{mmol}), \mathrm{Pd}_{2}(\mathrm{dba})_{3}(0.5$ mol \%), ligand $(2 \mathrm{~mol} \%)$, toluene $(3 \mathrm{~mL}), 10 \mathrm{~h}, 90{ }^{\circ} \mathrm{C}$. ${ }^{b}$ Conversion determined by inspection of the corresponding ${ }^{1} \mathrm{H}$ NMR spectra of crude reaction isolates. See the Supporting Information for details. ${ }^{e}$ Meaning derived from naphthyl azide.

$$
\begin{gathered}
\text { Gevorgyan } \\
\text { and co-workers }
\end{gathered}
$$

Figure 6. Electrostatic potential charges as determined by Gevorgyan and co-workers, ${ }^{19}$ indicates rationale for selective C-5 deprotonation.

Scheme 6. Trimethylsilylacetylene (23c) and Aryl Azides (24a-i) React under Desilylative CuAAC Reaction Conditions to Deliver 1-Substituted 1,2,3-Triazoles (28a-i)

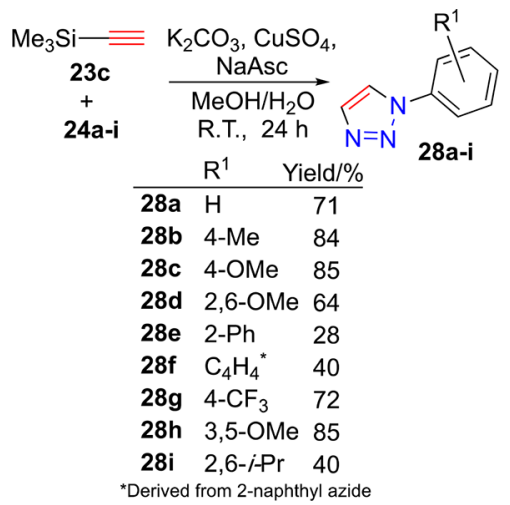

employed under a similar protocol, with varying amounts of $n$ butyllithium. In all cases, the same 5-phosphino-triazole product, 18a, was obtained, shown in Scheme 2. In the case of 22a, it was possible to isolate small quantities of the desired 
Scheme 7. Deprotonation of 1-Aryl 1,2,3-Triazole Derivatices and Subsequent Reaction with Phosphorous Chloride Reagent Protocol for Delivery of $29 a-n$

\begin{tabular}{|c|c|c|c|}
\hline 28a-i & $\begin{array}{l}\text { i, n-BuLi, } \\
-78^{\circ} \mathrm{C}, 2 \mathrm{~h} ; \\
\mathrm{ii}, \mathrm{CIP}\left(\mathrm{R}^{2}\right)_{2} \\
-78^{\circ} \mathrm{C}, 10 \mathrm{~h} \\
\mathrm{THF}\end{array}$ & $R^{2}{ }^{2}-R^{R}$ & $\begin{array}{ll}R^{1} \\
=\end{array}$ \\
\hline & $\mathrm{R}^{1}$ & $\mathrm{R}^{2}$ & Yield $/ \%$ \\
\hline $29 a$ & $\mathrm{H}$ & $\mathrm{Ph}$ & 47 \\
\hline $29 b$ & $\mathrm{H}$ & Сy & 73 \\
\hline $29 c$ & 4-Me & $\mathrm{Ph}$ & 36 \\
\hline 29d & 4-OMe & $\mathrm{Ph}$ & 49 \\
\hline $29 e$ & 2,6-OMe & $\mathrm{Ph}$ & 82 \\
\hline $29 f$ & 2,6-OMe & $\dot{i P r}$ & 88 \\
\hline $29 \mathrm{~g}$ & 2,6-OMe & Cy & 73 \\
\hline $29 h$ & 2,6-OMe & $t$-Bu & 72 \\
\hline $29 i$ & 2-Ph & $\mathrm{Ph}$ & 27 \\
\hline 29j & $\mathrm{C}_{4} \mathrm{H}_{4}{ }^{*}$ & $\mathrm{Ph}$ & 37 \\
\hline $29 k$ & $4-\mathrm{CF}_{3}$ & $\mathrm{Ph}$ & 68 \\
\hline 291 & 3,5-OMe & $\mathrm{Ph}$ & 72 \\
\hline $29 m$ & $2,6-i-\operatorname{Pr}$ & Cy & 89 \\
\hline $29 n$ & $2,6-i-\mathrm{Pr}$ & $t$-Bu & 88 \\
\hline
\end{tabular}

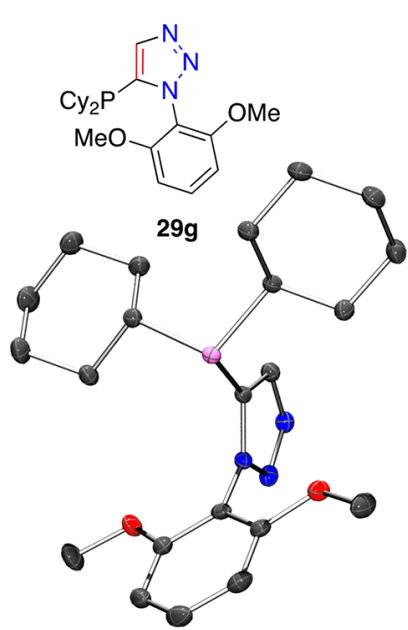

Figure 7. Representation of the crystal structure of $29 \mathrm{~g}$, ellipsoids drawn at the $50 \%$ probability level (Ortep3 for Windows and PovRay), hydrogen atoms omitted for clarity.

product 17a. X-ray crystal structures of both $17 \mathbf{a}$ and $18 \mathrm{a}$ were determined as shown in Figure 5.

In fact these observations should not have been at all unanticipated. ${ }^{102,104}$ The aforementioned report of Balakrishna and co-workers had previously probed the reaction of $17 \mathbf{a}$ in more detail than us under analogous conditions ${ }^{102}$ and determined a "kinetic" and "thermodynamic" relationship between lithium-halogen exchange alone, versus lithiumhalogen exchange followed by lithium (triazole-) proton exchange leading to products $17 \mathbf{a}$ and $18 \mathbf{a}$ respectively, Scheme 3.

Attempts to block the triazole $5-\mathrm{H}$ position, using the deuterium masking approach deployed by Richards and coworkers in the preparation of ferrocene derivatives, ${ }^{119}$ did not dramatically modify reaction outcomes in our hands. Variously deuterated products were always obtained from attempted lithiation-mediated access to products under routine conditions.

Since the scope of 18a-like ligands had not been investigated beyond the three triazole backbones reported by Glover et
Table 2. Ligand Screening: 1-Substituted 1,2,3-TriazoleContaining Phosphine Ligand Mediated, PalladiumCatalyzed, Reaction of Arylbromide 25 in the Formation of 27

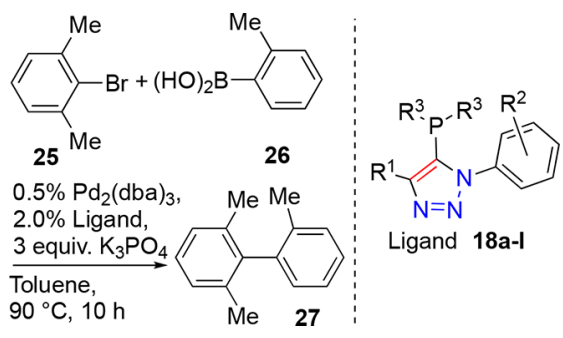

\begin{tabular}{clllc} 
entry & \multicolumn{1}{c}{$\mathrm{R}^{1}$} & \multicolumn{1}{c}{$\mathrm{R}^{2}$} & \multicolumn{1}{c}{ ligand } & conversion $[\%]^{a, b}$ \\
$\mathbf{1}$ & $\mathrm{H}$ & $\mathrm{Ph}$ & $\mathbf{2 9 a}$ & 20 \\
$\mathbf{2}$ & $\mathrm{H}$ & $\mathrm{Cy}$ & $\mathbf{2 9 b}$ & 98 \\
$\mathbf{3}$ & $4-\mathrm{Me}$ & $\mathrm{Ph}$ & $\mathbf{2 9 c}$ & 54 \\
$\mathbf{4}$ & $4-\mathrm{OMe}$ & $\mathrm{Ph}$ & $\mathbf{2 9 d}$ & 28 \\
$\mathbf{5}$ & $2,6-\mathrm{OMe}$ & $\mathrm{Ph}$ & $\mathbf{2 9 e}$ & 90 \\
$\mathbf{6}$ & $2,6-\mathrm{OMe}$ & $i-\mathrm{Pr}$ & $\mathbf{2 9 f}$ & 99 \\
7 & $2,6-\mathrm{OMe}$ & $\mathrm{Cy}$ & $\mathbf{2 9 g}$ & 99 \\
$\mathbf{8}$ & $2,6-\mathrm{OMe}$ & $t-\mathrm{Bu}$ & $\mathbf{2 9 h}$ & 98 \\
$\mathbf{9}$ & $2-\mathrm{Ph}$ & $\mathrm{Ph}$ & $\mathbf{2 9 i}$ & 82 \\
$\mathbf{1 0}$ & $\mathrm{C}_{4} \mathrm{H}_{4}{ }^{c}$ & $\mathrm{Ph}$ & $\mathbf{2 9 j}$ & 29 \\
$\mathbf{1 1}$ & $4-\mathrm{CF} \mathrm{F}_{3}$ & $\mathrm{Ph}$ & $\mathbf{2 9 k}$ & 83 \\
$\mathbf{1 2}$ & $3,5-\mathrm{OMe}$ & $\mathrm{Ph}$ & $\mathbf{2 9 l}$ & 51 \\
$\mathbf{1 3}$ & $2,6-i-\mathrm{Pr}$ & $\mathrm{Cy}$ & $\mathbf{2 9 m}$ & 99 \\
$\mathbf{1 4}$ & $2,6-i-\mathrm{Pr}$ & $t-\mathrm{Bu}$ & $\mathbf{2 9 n}$ & 99 \\
$\mathbf{1 5}$ & & & $\mathrm{S}-\mathrm{Phos}(\mathbf{7})$ & 98 \\
$\mathbf{1 6}$ & & & $\mathrm{X}-\mathrm{Phos}(\mathbf{8})$ & 99
\end{tabular}

${ }^{a}$ Reaction conditions: 2 -bromo- $m$-xylene $(0.4 \mathrm{mmol}), o$-tolylboronic acid $(0.6 \mathrm{mmol})$, potassium phosphate $(1.2 \mathrm{mmol}), \mathrm{Pd}_{2}(\mathrm{dba})_{3}(0.5$ $\mathrm{mol} \%)$, ligand $(2.0 \mathrm{~mol} \%)$, toluene $(3 \mathrm{~mL}), 10 \mathrm{~h}, 90{ }^{\circ} \mathrm{C}$. ${ }^{b}$ Conversion determined by inspection of the corresponding ${ }^{1} \mathrm{H}$ NMR spectra of crude reaction isolates. See the Supporting Information for details. ${ }^{c}$ Derived from 2-naphthyl azide.

Table 3. Ligand Screening: 1-Substituted 1,2,3-TriazoleContaining Phosphine Ligand Mediated, PalladiumCatalyzed, Reaction of Arylbromide 30 in Formation of 32

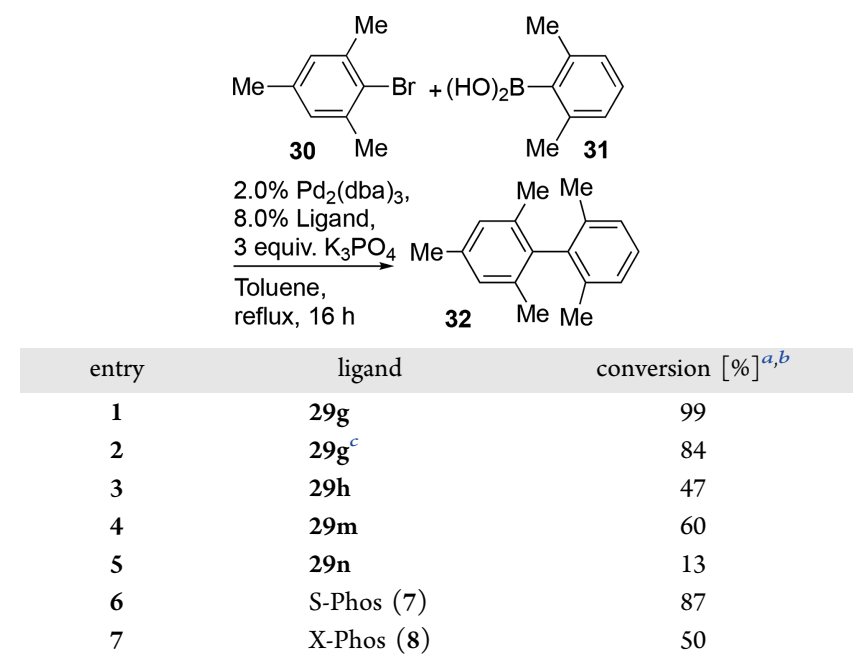

${ }^{a}$ Reaction conditions: 2-bromomesitylene (0.5 mmol), 2,6-xylylboronic acid $(1.0 \mathrm{mmol})$, potassium phosphate $(2 \mathrm{mmol}), \mathrm{Pd}_{2}(\mathrm{dba})_{3}(2.0$ mol \%), ligand $(8.0 \mathrm{~mol} \%)$, toluene $(3 \mathrm{~mL}), 18 \mathrm{~h}$, reflux. ${ }^{b}$ Determined by GC analysis with $n$-dodecane as internal standard. ${ }^{c} \mathrm{Pd}_{2}(\mathrm{dba})_{3}(0.5 \mathrm{~mol} \%)$, ligand $(2 \mathrm{~mol} \%)$. 


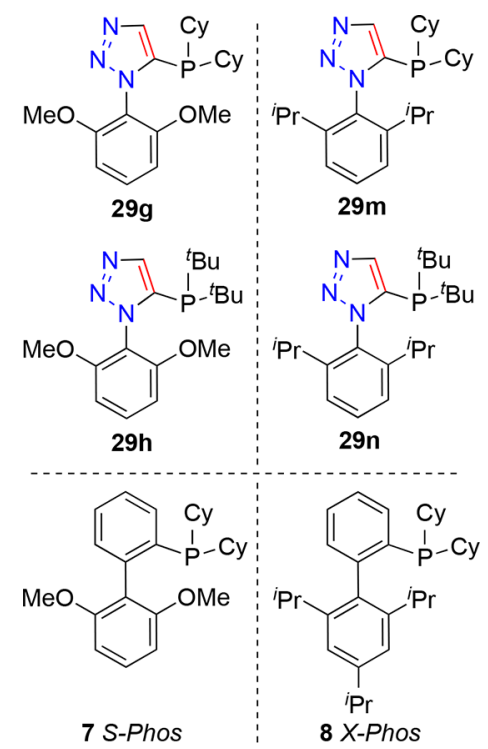

Figure 8. Ligands compared in Table 3 and Table 4.

Table 4. Ligand Screening: 1-Substituted 1,2,3-TriazoleContaining Phosphine Ligand-Mediated, PalladiumCatalyzed, Reaction of Arylchloride 37 in the Formation of 27

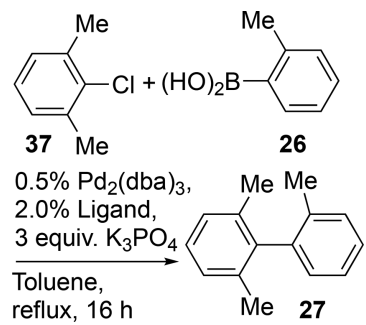

\begin{tabular}{clcc|} 
entry & \multicolumn{1}{c}{ ligand } & conversion $[\%]^{a, b}$ & isolated yield [\%] \\
$\mathbf{1}$ & $\mathbf{2 9 g}$ & 99 & 93 \\
$\mathbf{2}$ & $\mathbf{2 9 \mathbf { g } ^ { c }}$ & 99 & 92 \\
$\mathbf{3}$ & $\mathbf{2 9 h}$ & 99 & 82 \\
$\mathbf{4}$ & $\mathbf{2 9 m}$ & 99 & 92 \\
$\mathbf{5}$ & $\mathbf{2 9 n}$ & 70 & \\
$\mathbf{6}$ & S-Phos (7) & 33 & 25 \\
$\mathbf{7}$ & X-Phos (8) & 99 & 90
\end{tabular}

${ }^{a}$ Reaction conditions: 2 -chloro- $m$-xylene $(1.0 \mathrm{mmol}), o$-tolyboronic acid $(1.5 \mathrm{mmol})$, potassium phosphate $(3.0 \mathrm{mmol}), \mathrm{Pd}_{2}(\mathrm{dba})_{3}(0.5$ mol \%), ligand $(2.0 \mathrm{~mol} \%)$, toluene $(3 \mathrm{~mL}), 10 \mathrm{~h}, 90{ }^{\circ} \mathrm{C}$. ${ }^{b}$ Determined by GC analysis with $n$-dodecane as internal standard. ${ }^{c} \mathrm{Pd}_{2}(\mathrm{dba})_{3}(0.25 \mathrm{~mol} \%)$, ligand $(1.0 \mathrm{~mol} \%)$.

al. ${ }^{104}$ and Choubey et al., ${ }^{102}$ we chose to focus attention on triazole 5-H lithiation to deliver a range of potential ligands for cross-coupling catalysis. To this end, alkynes $23 \mathbf{a}$ and $23 \mathbf{b}$ reacted smoothly with azides $\mathbf{2 4 a - f}$ to furnish 1,5 disubstituted 1,2,3-triazoles $21 \mathbf{a}-\mathbf{i}$ in acceptable to good yields, shown in Scheme 4.

Applying the aforementioned triazole lithiation and subsequent quench with dicyclohexyl-, di-iso-propyl-, or diphenyl- phosphorus chloride reagent protocol to isolated 21a-i triazole set, with the express expectation of generating 5phosphino triazole derivatives, delivered 12 targeted phosphino-triazoles $18 \mathbf{a}-1$ in acceptable to good yields (Scheme 5).

In order to benchmark the catalytic capability of ligands in this report, the palladium-catalyzed Suzuki-Miyaura reaction
Table 5. Ligand Screening: 1-Substituted 1,2,3-TriazoleContaining Phosphine Ligand Mediate, PalladiumCatalyzed, Reaction of Arylchloride 37 in Formation of 32

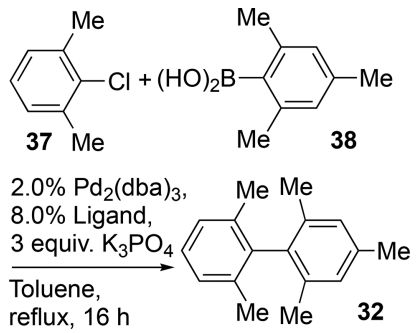

\begin{tabular}{clc} 
entry & \multicolumn{1}{c}{ ligand } & conversion $[\%]^{a, b}$ \\
$\mathbf{1}$ & $\mathbf{2 9 g}$ & 49 \\
2 & $\mathbf{2 9 m}$ & 37 \\
3 & S-Phos (7) & 55 \\
4 & X-Phos (8) & 5 \\
\hline
\end{tabular}

${ }^{a}$ Reaction conditions: 2 -chloro- $m$-xylene $(0.5 \mathrm{mmol})$, 2,4,6-trimethylphenylboronic acid $(1.0 \mathrm{mmol})$, potassium phosphate $(2 \mathrm{mmol})$, $\mathrm{Pd}_{2}(\mathrm{dba})_{3}(2.0 \mathrm{~mol} \%)$, ligand $(8.0 \mathrm{~mol} \%)$, toluene $(3 \mathrm{~mL}), 18 \mathrm{~h}$, reflux. ${ }^{b}$ Determined by GC analysis with $n$-dodecane as internal standard.

Scheme 8. Percentage Homo-Coupled Product 36 under Described Reaction Conditions Using 50\% Excess Boronic Acid 34

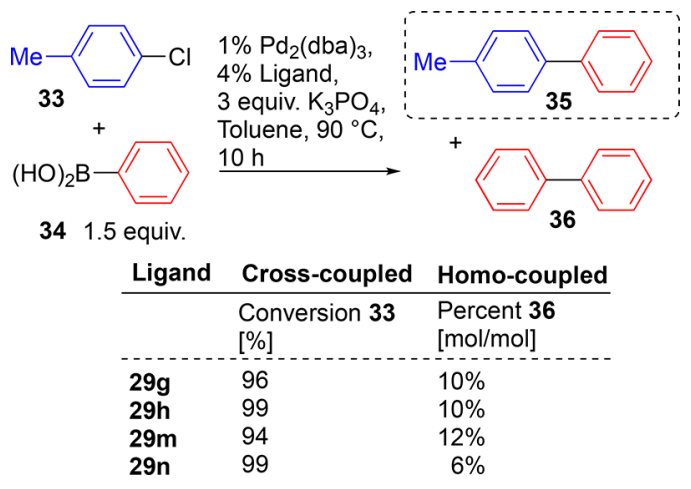

of 2-bromo-m-xylene (25) and ortho-tolylboronic acid (26) under standard conditions ( $1 \mathrm{~mol} \%$ palladium, $2 \mathrm{~mol} \%$ ligand, three equivalents of base, $10 \mathrm{~h}$, toluene, $90{ }^{\circ} \mathrm{C}$, see the Supporting Information) was compared. The catalyzed formation of compound 27 represents a challenging but achievable cross-coupling: While an aryl bromide is employed in the reaction, the product (a triply ortho-substituted biaryl) is sterically congested about the formed bond. Diphenyl aryl phosphine 17a and 5-phosphino triazoles 18a-1 were employed as ligands in the benchmark reaction (Table 1). The use of $17 \mathbf{a}$ as ligand (Table 1 , entry 1 ) resulted in $29 \%$ conversion to product 27 . The 5-phosphino isomer of $17 \mathbf{a}$, 18a, also gave less than 50\% conversion to desired product 27 in the same reaction (Table 1 , entry 2). As may be expected, switching the diphenylphosphine part of 18a to dialkylphosphine groups di-iso-propyl (18b) and dicyclohexyl (18c) improved the reaction outcomes, resulting in 62 and $75 \%$ conversion, respectively (Table 1 , entries 3 and 4). Changing the alkyne-derived part of the triazole from phenyl (18a) to cyclohexyl (18d) gave a marked improvement delivering product 27 in $86 \%$ conversion (Table 1, entry 2 versus 5 ). 

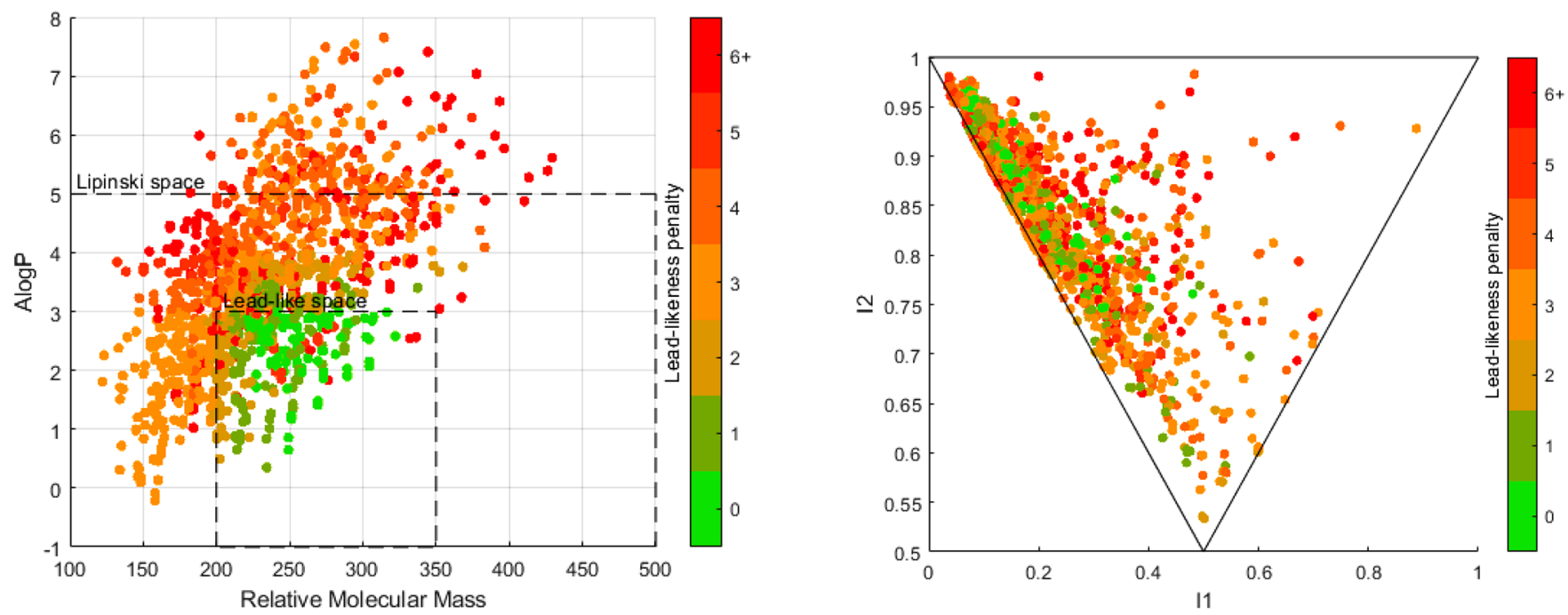

Figure 9. Virtual Suzuki-Miyaura catalysis products generated and analyzed in the LLAMA web tool. Left: $A$ log $P$ versus molecular mass, Lipinski and lead-like space indicated. Right: PMI plot, rod, disc, sphere axis. (See the Supporting Information for data tables.)

While good results were obtained in the Suzuki-Miyaura cross-coupling reactions with 18-derived catalysts the more effective ligands (generally larger) suffered somewhat from poor solubility. Thus, ligand modifications that retained activity but allowed for more ready synthesis and manipulation at larger scale were sought. From briefly surveying the results in Table 1 it was concluded that changes in the $\mathrm{R}^{1}$ (alkynederived) part (e.g., entry 8 versus entry 9) were less influential on the reaction outcome than changes in the $\mathrm{R}^{2}$ (azidederived) part (e.g., entry 5 versus entry 13). Reasoning that smaller ligands may benefit from enhanced solubility and tractability, a strategy to retain the $\mathrm{N}$-substituents (azidederived parts) while minimizing the alkyne-derived parts was chosen for further elaboration. Gevorgyan and co-workers have already reported that 1,5-disubstituted 1,2,3-triazoles may be accessed by selective reaction at the 5-position of 1-substituted triazoles (Figure 6 shows the electrostatic potentials of the triazole-carbons they determined), and coupled with the synthetic strategies reported by Oki et al. ${ }^{103}$ for chiral bisphosphine synthesis, this led to the conclusion that 1substitutued triazoles may be readily converted to a library of 1-substituted, 5-phosphino 1,2,3-triazoles

Following the optimized protocol of Oki et al. ${ }^{103}$ deployed in the synthesis of more complex constructs, a range of 1substituted triazoles were synthesized. Specifically, trimethylsilylacetylene $(\mathbf{2 3 c})$ and aryl azides $(\mathbf{2 4 a}-\mathbf{i})$ were exposed to $\mathrm{CuAAC}$ reaction conditions (Scheme 6) that led to effective triazole formation and desilylation in one pot. Acceptable to good yields of 1 -substituted triazoles $28 \mathbf{a}-\mathbf{i}$ were isolated after $24 \mathrm{~h}$ room temperature reactions.

Triazoles $28 \mathbf{a}-\mathbf{i}$ reacted smoothly under the deprotonation and phosphorus chloride reagent quench reaction conditions described earlier. Deprotonation at $-78{ }^{\circ} \mathrm{C}$, by treatment with $n$-butyllithium, followed by addition of dicyclohexyl-, di-isopropyl-, di-tert-butyl- or diphenyl-phosphorus chloride at the same temperature (Scheme 7) resulted in formation of the desired triazole-containing phosphines $\mathbf{2 9 a}-\mathbf{n}$ in acceptable to good yields. The X-ray crystal structure of $\mathbf{2 9} \mathrm{g}$ was determined (Figure 7); the orientation of the molecule (in the solid state) is such that the lone pair of the phosphine is oriented to the same direction as the 1-aryl substituent of the triazole. In turn, this orientation about a central five-membered ring describes a relatively wide binding pocket for metals with potential for arene-metal interactions alongside primary phosphorus-metal ligation.

Ligands $29 \mathbf{a}-\mathbf{n}$ were tested in the aforementioned benchmark Suzuki-Miyaura cross-coupling reaction of 25 with 26 catalyzed by a palladium-phosphine complex, to produce biaryl 27 (Table 2, entries 1-14). Under the same conditions, commercially sourced ligands, S-Phos (7) and X-Phos (8) were also used for comparison (Table 2, entries 15 and 16, respectively). Diphenyl-phosphino triazole derived ligands failed to deliver product $\mathbf{2 7}$ in good yields, under the conditions employed the best conversion for this ligand class was only $54 \%$ (ligand 29c, Table 2, entry 3 ), whereas dialkylphosphino triazoles gave universally excellent conversion, equal to the commercially sourced S- and X-Phos in performance, under these conditions.

While pleased to have created ligands offering good performance in a benchmark reaction, the reaction itself did not offer enough diversity of outcomes to evaluate dialkylphosphino ligand performances against each other nor against readily available commercial ligands 7 and 8 . Next, a more sterically demanding test-reaction was chosen to evaluate the ligands further. The formation of biaryl $\mathrm{C}-\mathrm{C}$ bonds where the formed bond is flanked by four ortho-substituents presents a particularly challenging yet attractive transformation, not in the least due to the apparent three-dimensional nature of the crosscoupled products. ${ }^{117}$ The reaction of bromide $\mathbf{3 0}$ with boronic acid 31 was selected as one such reaction to probe catalyst effectiveness (Table 3). Conversion to product 32 may be monitored by gas chromatographic analysis, facilitating ready comparison of reactions performed in parallel. Having given quantitative conversions to product 27 (Table 2, entries 7, 8, 13 , and 14), and being both relatively easy to synthesize and available in sufficient quantities, ligands $29 \mathrm{~g}, 29 \mathrm{~h}, 29 \mathrm{~m}$, and 29n were selected for further investigation. These ligands are triazole-analogues of leading phenylene ligands 7 and 8; thus, in order to probe any specific advantages of triazole-core ligands they were compared directly against S-Phos and XPhos phenylene ligands. (For retained and compared ligands, see Figure 8.) 
Scheme 9. Microwave-Heated Synthesis $(1 \mathrm{~h})$ of 10 Leadlike Compounds by Suzuki-Miyaura Cross-Coupling Reactions Using $2 \mathrm{~mol} \%$ Palladium and $4 \mathrm{~mol} \%$ Ligand $29 \mathrm{~g}$

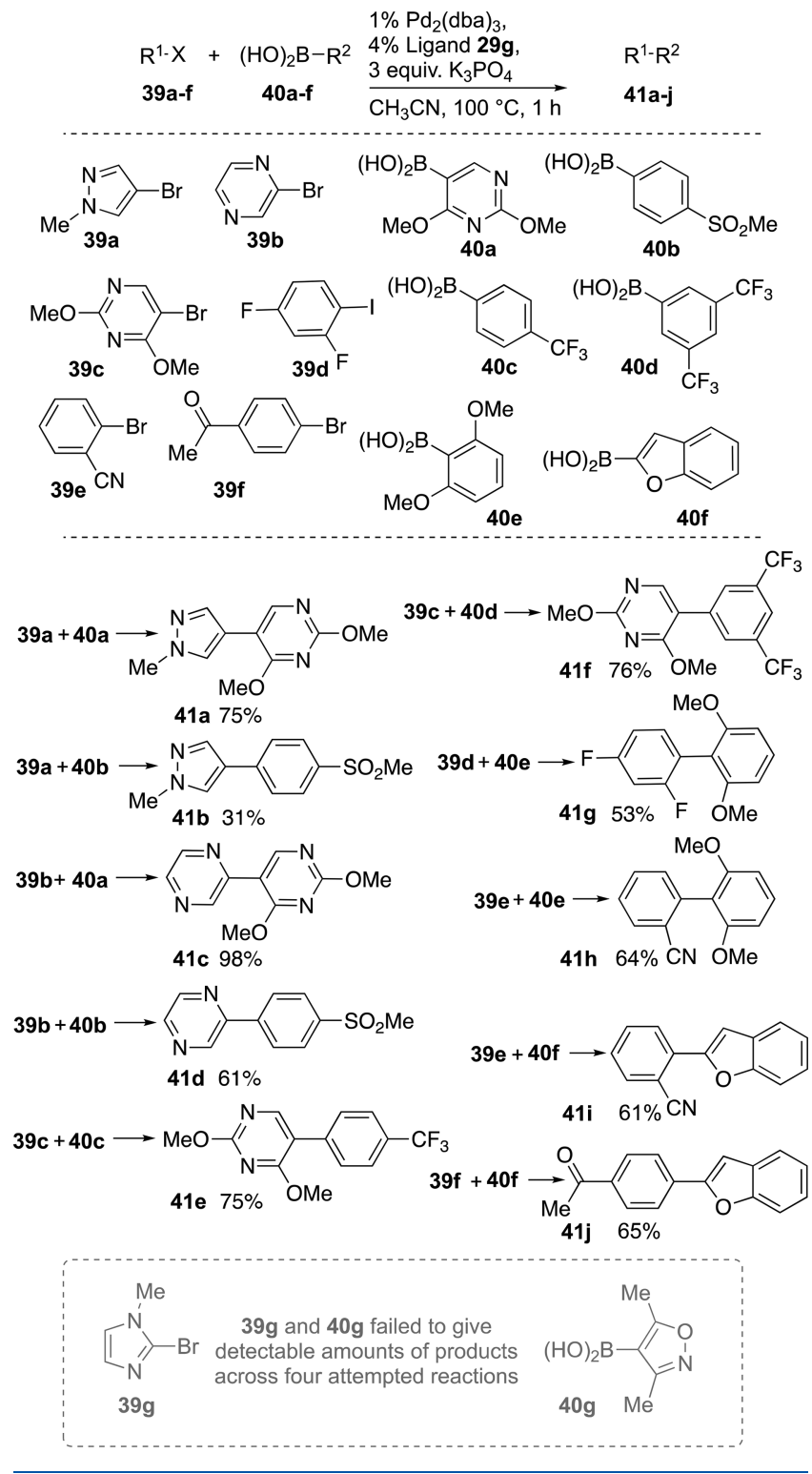

In order to ensure good reaction conversions, the catalyst loading, temperature, and reaction time were all increased in comparison to the earlier Suzuki-Miyaura reactions. The standard conditions employed in the comparisons of Table 3 (entries 1 and 3-7) were $4 \mathrm{~mol} \%$ palladium and $8 \mathrm{~mol} \%$ ligand in toluene at reflux for $16 \mathrm{~h}$. Under these conditions, cyclohexyl-substituted triazole-containing ligands $29 \mathrm{~g}$ and $29 \mathrm{~m}$ gave higher conversions to 32 than did their tert-butylsubstituted analogues $29 \mathrm{~h}$ and 29n (Table 3, entries 1 and 4 (99 and 60\%) versus entries 3 and 5 (47 and 13\%), respectively). In this comparison, the use of S-Phos (7) as ligand gave $87 \%$ conversion (Table 3 , entry 6 ) and the use of $\mathrm{X}$-Phos (8) as ligand gave $50 \%$ conversion (Table 3, entry 7 ) to compound 32 (under these conditions). Since ligand $\mathbf{2 9 g}$ gave the best conversion (under the conditions employed), catalyst loading was reduced to $1 \mathrm{~mol} \%$ palladium (in the form of $\left.0.5 \mathrm{~mol} \% \mathrm{Pd}_{2}(\mathrm{dba})_{3}\right)$ alongside $2 \mathrm{~mol} \% 29 \mathrm{~g}$ as ligand
(Table 3, entry 2), and under these conditions, a conversion of $84 \%$ to 32 was achieved.

To further probe the utility of 1-aryl 5-phosphino 1,2,3triazoles as ligands in Suzuki-Miyaura catalysis the synthesis of compound $\mathbf{2 7}$ from aryl chloride 37 and boronic acid 26 was investigated (shown in Table 4) deploying the same ligand set as in Table 3. The reaction conditions mirrored those used earlier for cross-coupling with bromide analogue 25 in Table 2, but in order to ensure good reaction conversions the reaction temperature was increased slightly (toluene at reflux).

Under the reaction conditions employed (Table 4), catalysts derived from triazole-containing ligands $29 \mathrm{~g}, 29 \mathrm{~h}$, and $29 \mathrm{~m}$ delivered compound $\mathbf{2 7}$ in quantitative yield (Table 4, entries 1,3 , and 4 , respectively). Using $\mathbf{2 9 g}$ as ligand at a lower catalyst loading of $0.5 \mathrm{~mol} \%$ of palladium proportionally, quantitative conversion to 27 (isolated yield 92\%) was achieved. In this comparison, the use of S-Phos (7) as ligand gave $33 \%$ conversion (Table 4 , entry 6 ), and the use of X-Phos (8) as ligand gave quantitative conversion (Table 4, entry 7 ) to compound 27.

Next, a demanding palladium-catalyzed reaction between 2chloro-meta-xylene (37) and 2,4,6-trimethylphenylboronic acid (38) leading to product 32 was attempted. Two triazole ligand-based catalyst systems were compared against catalyst systems derived from S-Phos (7) and X-Phos (8); see Table 5.

Under the conditions employed, the four catalyst systems compared produced only moderate yields. That is not to say that these reactions could not be optimized further, but the side-by-side comparison revealed S-Phos (7) to be slightly better than triazole-containing phosphine $29 \mathrm{~g}$ (55 versus $49 \%$ conversion; Table 5, entry 4 versus entry 1 , respectively). Slightly lower conversions were obtained when $29 \mathrm{~m}$ or X-Phos were used as ligands (Table 5 entries 2 and 4 respectively).

One potential problem with Suzuki-Miyaura catalyzed reactions, particularly evident when using less reactive aryl chlorides in cross-coupling, is homocoupling of the boronic acid containing reaction partner. ${ }^{120}$ In order to test our routine reaction protocols and our four selected triazole ligands $(\mathbf{2 9 g}$, 29h, 29m, and 29n) for their propensity to lead to undesired homocoupled product, the following reaction was probed. Aryl chloride 33 was reacted with 1.5 equiv of phenyl boronic acid 34. Catalyst loading was $2 \mathrm{~mol} \%$ palladium and $4 \mathrm{~mol} \%$ ligand. The reactions were conducted in toluene at $90{ }^{\circ} \mathrm{C}$ with 3 equiv of potassium phosphate as base; see Scheme 8. Set up like this, we can judge a reaction to be successful, i.e., not suffering from an adventitious homocoupling side reaction leading to product composition, if conversion of aryl chloride 33 is high (near 100\%) and the amount of formed byproduct 36 is low. Choosing 1.5 equiv of boronic acid gives a chance for the formation of 36 , thus evidencing the cross- versus homo-coupling potential of the ligands in the chosen SuzukiMiyaura reaction under the conditions described.

All four of the tested ligands (Scheme 8, 29g, 29h, 29m, and 29n) gave good conversion to product 35 (ligands $29 \mathrm{~h}$ and 29n leading to complete consumption of stating aryl chloride 33). In this case the apparently most bulky ligand, 29n, performed best giving just $6 \%(\mathrm{~mol} / \mathrm{mol})$ homocoupled product (36); the other three ligands gave rise to only slightly elevated amounts of homocoupled side product (10-12\% $(\mathrm{mol} / \mathrm{mol}))$. Thus, demonstrating that under the conditions employed, reaction protocols used throughout this study do not suffer appreciably from loss of halide-containing starting materials through unwanted homocoupling. 

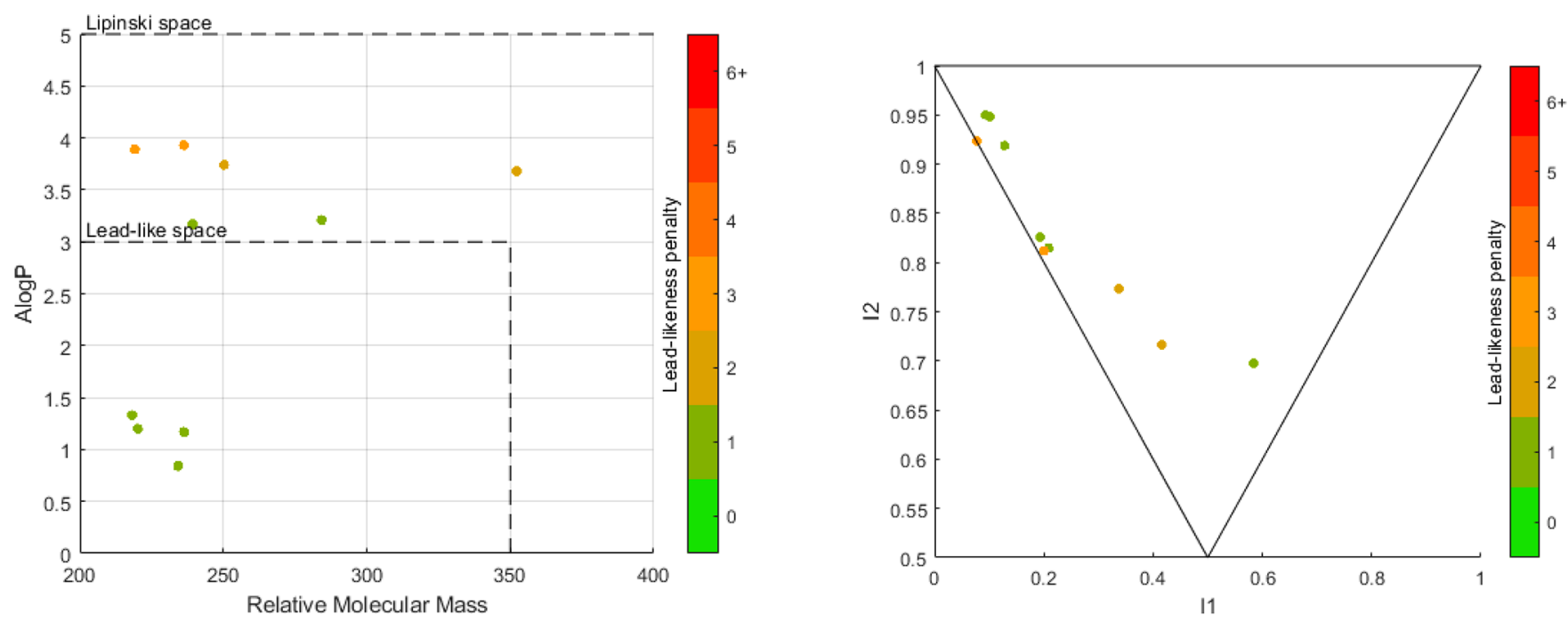

Figure 10. Left: $M_{\mathrm{w}}$ vs $A \log P$. Right: PMI analysis of products synthesized in Scheme 9.

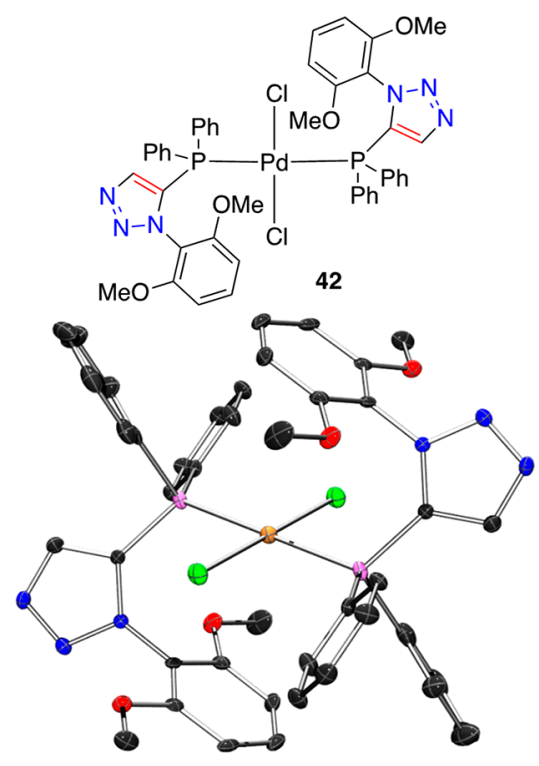

Figure 11. Representation of the crystal structure of $\mathbf{4 2}$, ellipsoids are drawn at the $50 \%$ probability level (Ortep3 for Windows and PovRay). The structure contains a palladium complex, which is located on an inversion center and two molecules of dichloromethane per complex. Only half of the complex and one dichloromethane molecule are unique. Pd...P bond lengths 2.322(9) Å. 2,6-Bismethoxy aryl-centroid...Pd $3.843(2) \AA$ A. Symmetry code used to generate equivalent atoms: $1-x,-y,-z$.

Lead-like Compounds. While the results discussed thus far have exemplified the effectiveness of ligands $29 \mathrm{~g}$ and $29 \mathrm{~m}$ to catalyze sterically demanding cross-coupling reactions, the ability to catalyze the cross-coupling of functionalities relevant to medicinal chemistry, to give lead- and drug-like products remains a critical need in the agrochemical and pharmaceutical sectors. $^{77}$ To this end, a range of bis-aromatic products, containing motifs of the type that are commonly encountered in medicinal chemistry, ${ }^{121-123}$ were identified, and their synthesis embarked upon. The products of virtual SuzukiMiyaura cross-couplings of 48 aromatic halides (iodides, bromides, or chlorides) and 44 aromatic boronic acids (or esters) from (i) a collection held within the lead research group; (ii) those curated within the University of Birmingham

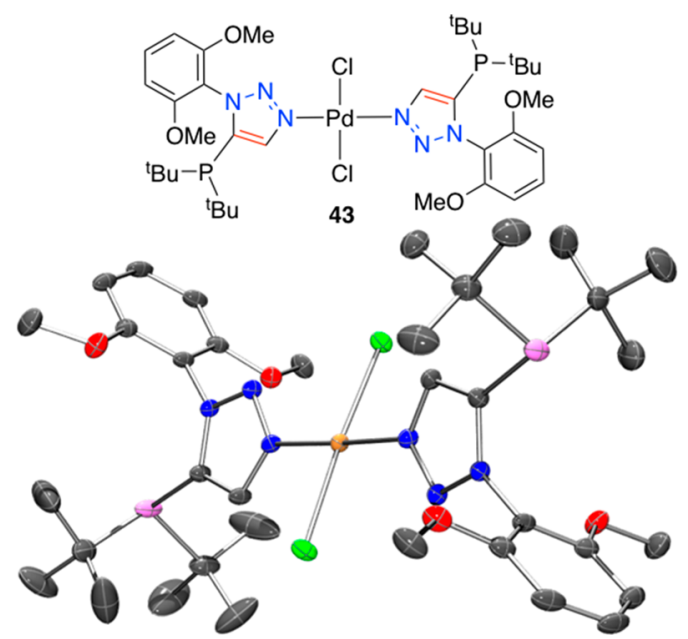

Figure 12. Representation of the crystal structure of 43, ellipsoids are drawn at the $50 \%$ probability level (Ortep3 for Windows and PovRay). The structure contains two molecules of dichloromethane per palladium complex (omitted for clarity).

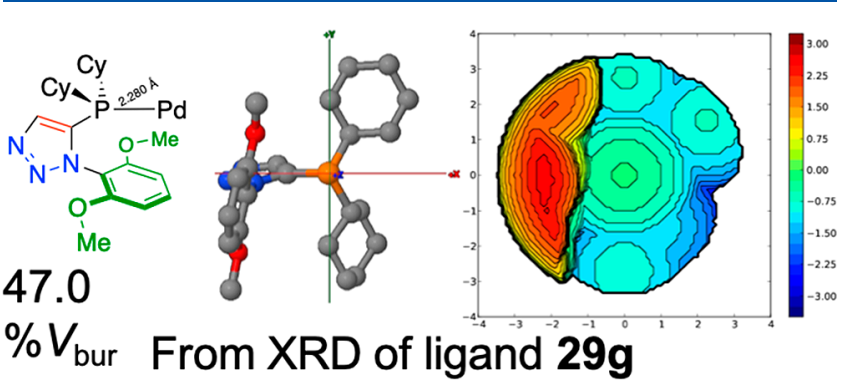

Figure 13. Steric map of phosphine-palladium complex: Derived from crystal structure of free ligand $29 \mathrm{~g}$ with a P-Pd distance of $2.280 \AA$ applied, resulting in a $47.0 \% V_{\text {bur }}$.

Scaffold Diversification Resource; ${ }^{124}$ and (iii) drawn from a boronic acid collection via the GSK Free Building Blocks resource; were enumerated and analyzed by the online resource LLAMA (Figure 9). ${ }^{118}$

The open-access web tool LLAMA allows the user to conduct virtual reactions and analyze the virtual product library for molecular properties such as molecular weight, $A \log P$, and 


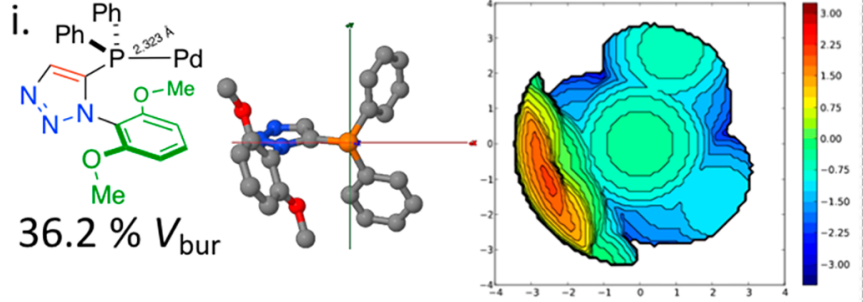

From XRD of $\mathbf{4 2}$ without adjusting $\mathrm{Pd}^{2+}-\mathrm{P}$ distance
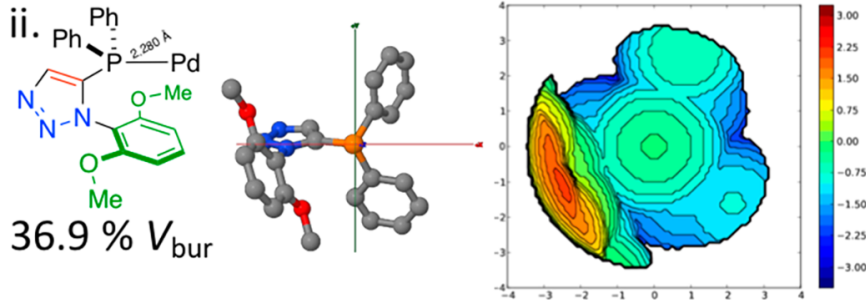

From XRD of $\mathbf{4 2}$ with adjustment of Pd-P to $2.280 \AA$

Figure 14. (i) Chemical structure of part of the crystallographically determined complex 42, with a 2.323(2) A Pd-P distance (from the crystal structure) used in the calculation of buried volume, $36.2 \% V_{\text {bur }}$ (ii) Chemical structure of the bond-length-modified, crystal-structure-informed palladium complex of ligand $29 \mathrm{e}$ in complex 42 (2.280 $\AA \mathrm{Pd}-\mathrm{P}$ distance was used in the buried volume calculation), $36.9 \% V_{\text {bur }}$.

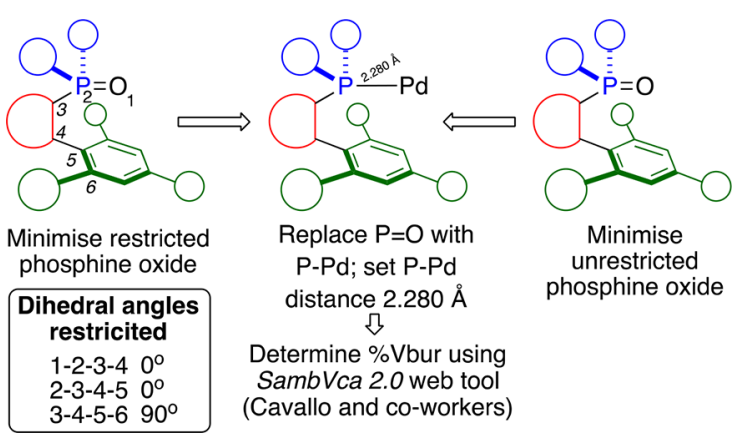

Figure 15. Protocol for obtaining structures for comparison of steric parameters via an unrestricted (right) and a dihedral angles restricted model (left). In both cases, a phosphine oxide model was minimized using Spartan' 16 and the $\mathrm{P}=\mathrm{O}$ later replaced with a $\mathrm{P}-\mathrm{Pd}$ bond of $2.280 \AA$. The structures thus obtained were then analyzed by the SambVca2.0 free web tool to determine the $\% V_{\text {bur }}$ and to create a steric map.

3D character. Figure 9 left shows the full virtual library of 1661 compounds created from the virtual Suzuki-Miyaura crosscoupling of the boronic acids and aryl halides described above. It shows that while the majority of these virtual products fall within Lipinkski space $\left(M_{\mathrm{w}}<500, A \log P<5\right)$ only $\sim 20 \%$ lie within lead-like space as defined by Churcher et al. $\left(200<M_{\mathrm{w}}\right.$ $<350, A \log P<3$ ). This fact is illustrated by the lead-likeness penalty scores of these compounds, which have an average of 3.47. This penalty scoring system was developed by the creators of LLAMA to visualize how far away from ideal leadlikeness a compound may be and incorporates all determined molecular properties into one score. Figure 9 (right side) shows the PMI analysis of the same 1661 virtual compounds in the library of virtual Suzuki-Miyaura cross-coupled products. A PMI plot describes the 3D shape of the lowest energy conformation of a compound on a triangular plot. The upper left corner represents rod-like compounds, the bottom corner represents disc-like compounds, and the upper right corner represents spherical compounds. This analysis shows that the majority of the virtual library resides close to the rod-disc axis, representing flat compounds.

From the 1661 virtual compounds constructed within the LLAMA tool, 14 possible products that accessed preferable lead-like chemical space and were selected for testing $\mathbf{2 9 g}$ mediated Suzuki-Miyaura cross-coupling reactions, as in Scheme 9. In this case, the screening conditions involved microwave heating in a sealed-tube at $100{ }^{\circ} \mathrm{C}$ in acetonitrile for just $1 \mathrm{~h}$. The possible products include challenging heteroatom-containing and/or ortho substituents, representing both a set of possible products displaying favorable characteristics for drug discovery and a robust challenge for road-testing our best new ligand, $\mathbf{2 9 g}$.

Pleasingly, most of the reactions attempted gave greater than $50 \%$ isolated yield of these challenging cross-coupled products $(41 \mathrm{a}-\mathbf{j})$; however, 2-bromo-1-methyl- $1 \mathrm{H}$-imidazole $39 \mathrm{~g}$ and (3,5-dimethylisoxazol-4-yl)boronic acid $40 \mathrm{~g}$ failed to deliver detectable amounts of desired cross-coupled products in four test scenarios under the conditions employed.

To determine the utility of the products created in this analysis, they were analyzed using the LLAMA web tool to determine their suitability as lead-like compounds (Figure 10). Figure 10 (left), shows how products $41 \mathbf{a}-\mathbf{j}$ explore the druglike space, with all products lying within the Lipinski space $\left(M_{\mathrm{w}}<500, A \log P<5\right)$ and a significant proportion lying within the lead-like space ( $40 \%$ of the synthesized com-

\section{Steric maps of phosphine-palladium complex of ligand} $29 \mathrm{e}$ - computed with selected dihedral angles locked
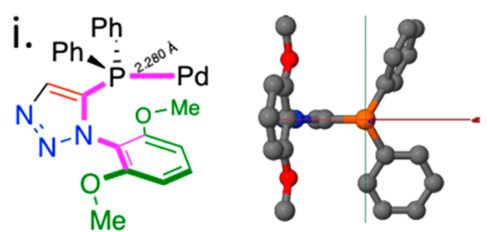

$44.2 \% V_{\text {bur }}$

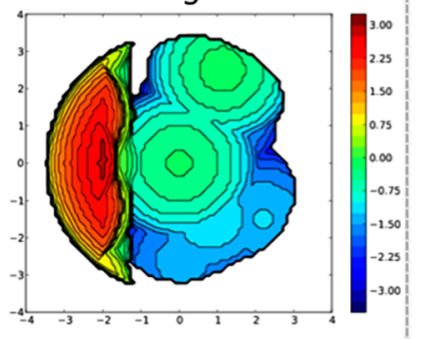

Steric maps of phosphine-palladium complex of ligand $29 \mathrm{e}$ - computed without rotation restriction
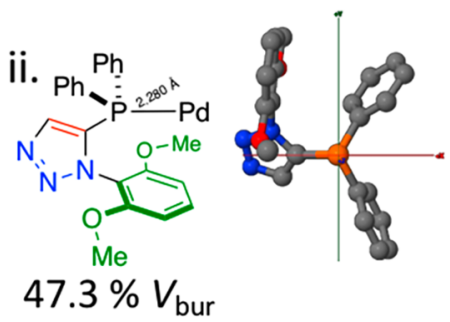

Figure 16. (i) Chemical structure and computationally determined steric map and buried volume, structures derived in silico and calculated with restricted dihedral angles as describe in Figure 15 (left), $44.2 \% V_{\text {bur }}$ (ii) Chemical structure and computationally determined steric map and buried volume of structures derived in silico and calculated without any dihedral restrictions as describe in Figure 15 (right), $47.3 \% V_{\text {bur }}$. 
a. Steric maps of phosphine-palladium complexes - selected dihedral angles locked

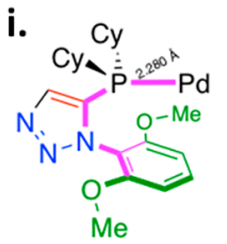

$45.5 \% V_{\text {bur }}$
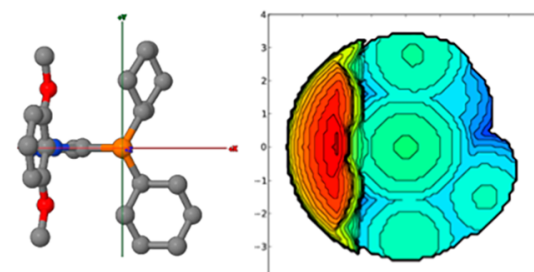

ii.

$47.8 \% V_{\text {bur }}$
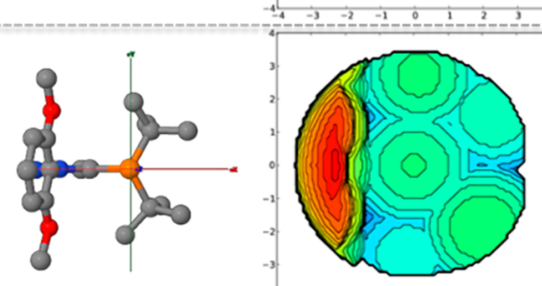

iii. $\mathrm{Cy}$

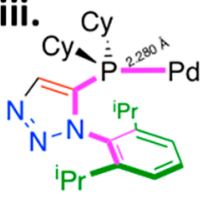

$47.1 \% V_{\text {bur }}$
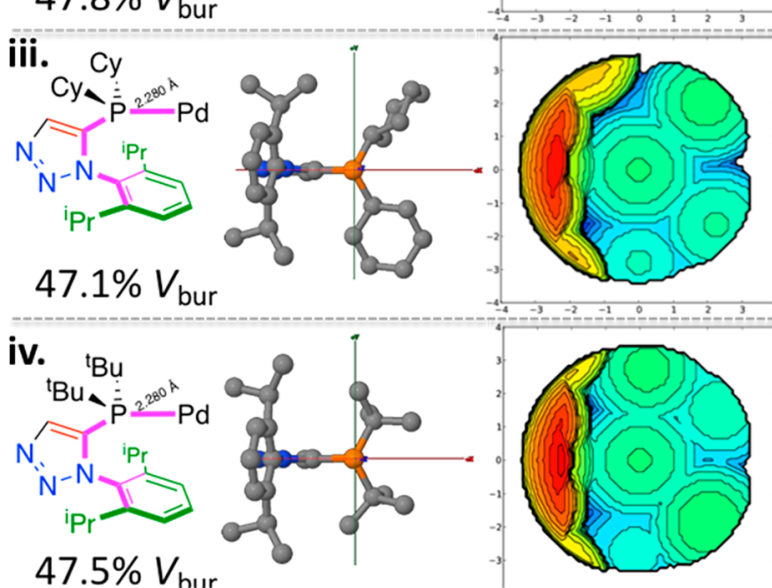

$47.5 \% V_{\text {bur }}$

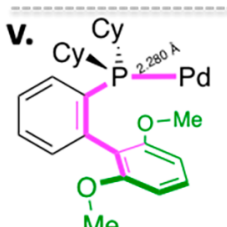

Me

$50.8 \% V_{\text {bur }}$
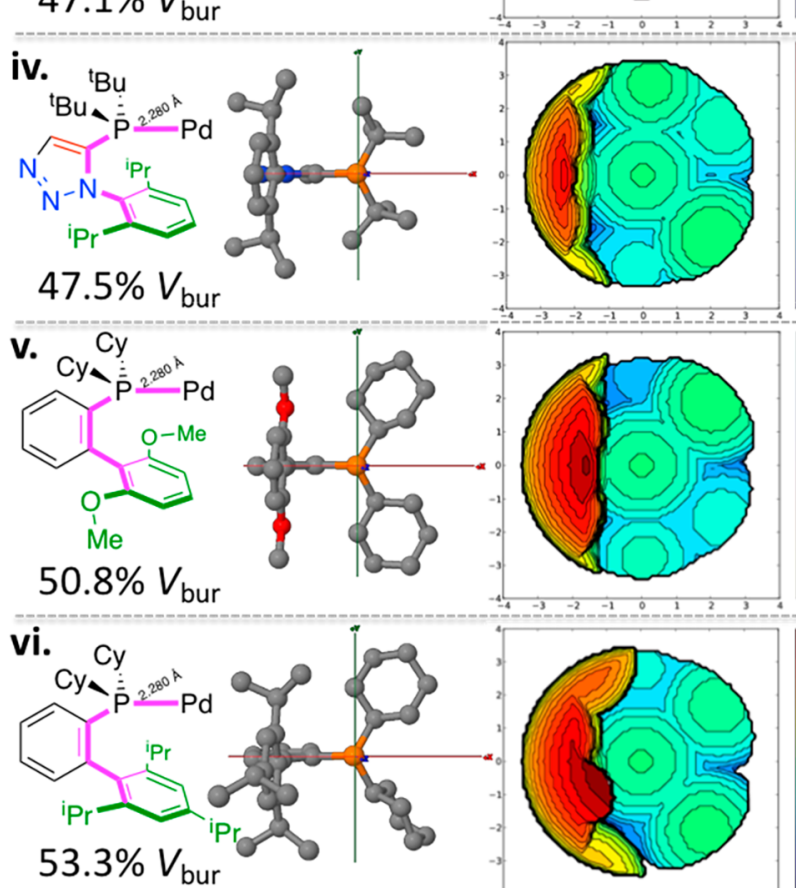

b. Steric maps of phosphine-palladium complexes - without rotation restriction
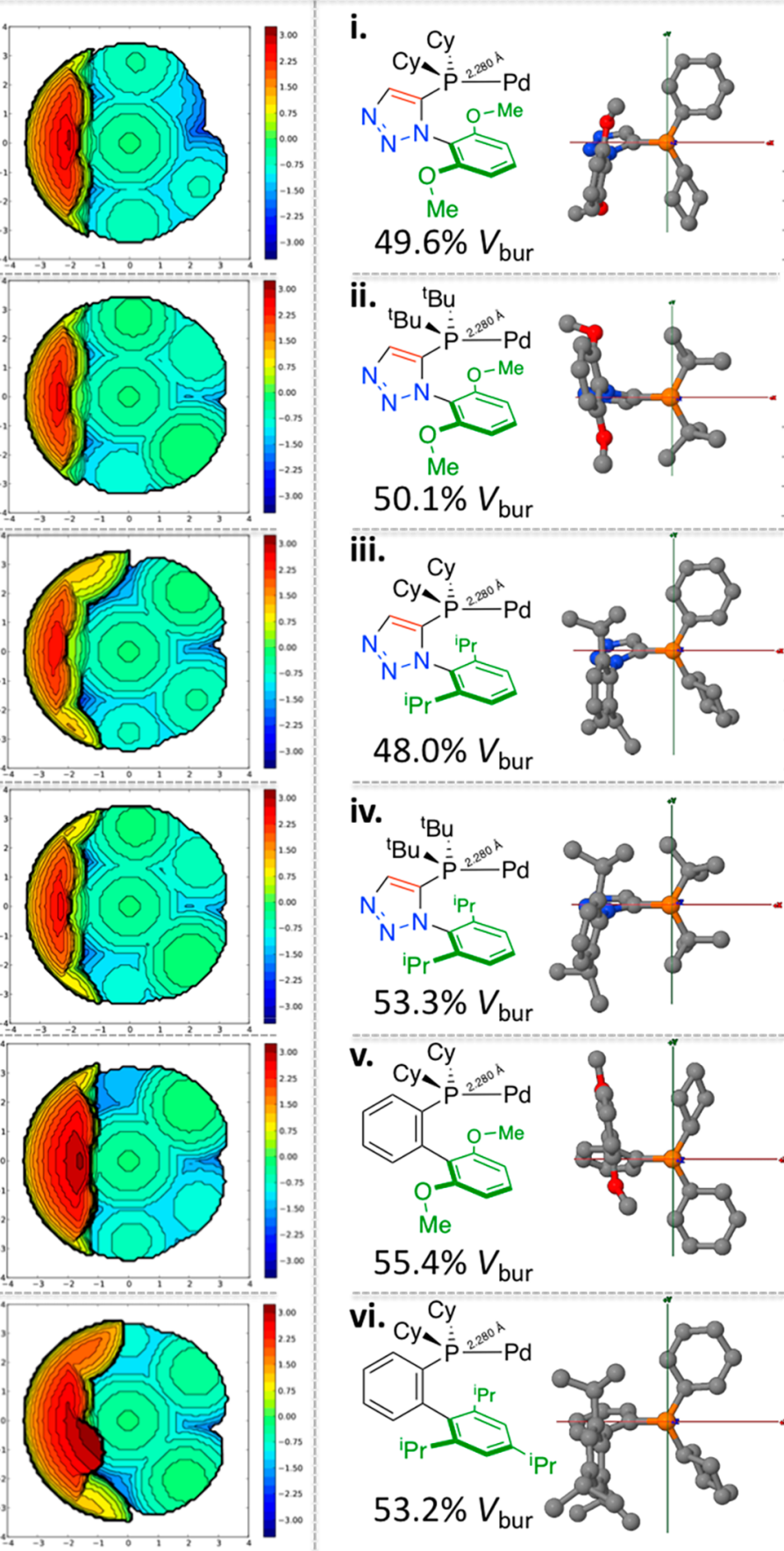

$49.6 \% V_{\text {bur }}$

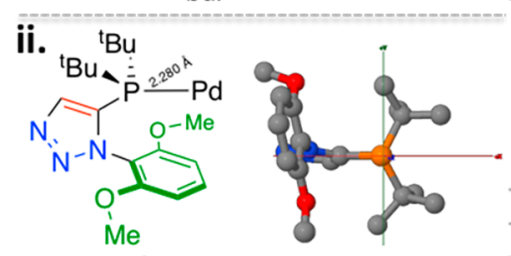

$50.1 \% V_{\text {bur }}$
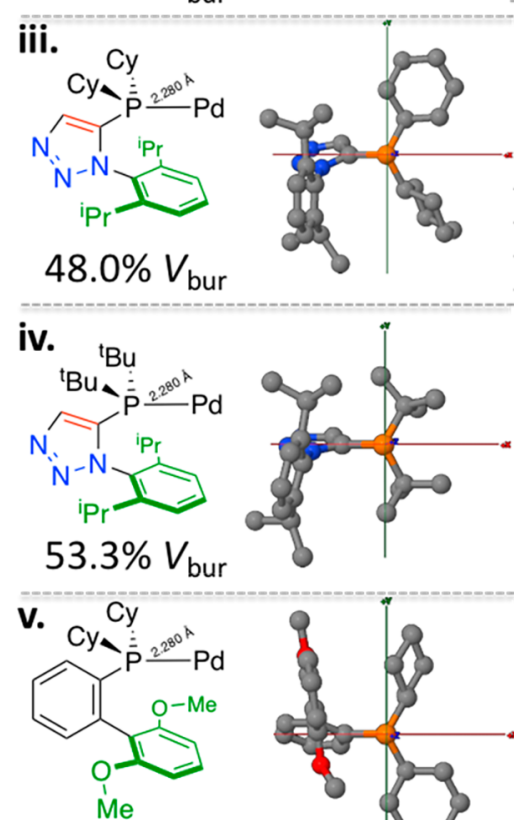

$55.4 \% V_{\text {bur }}$
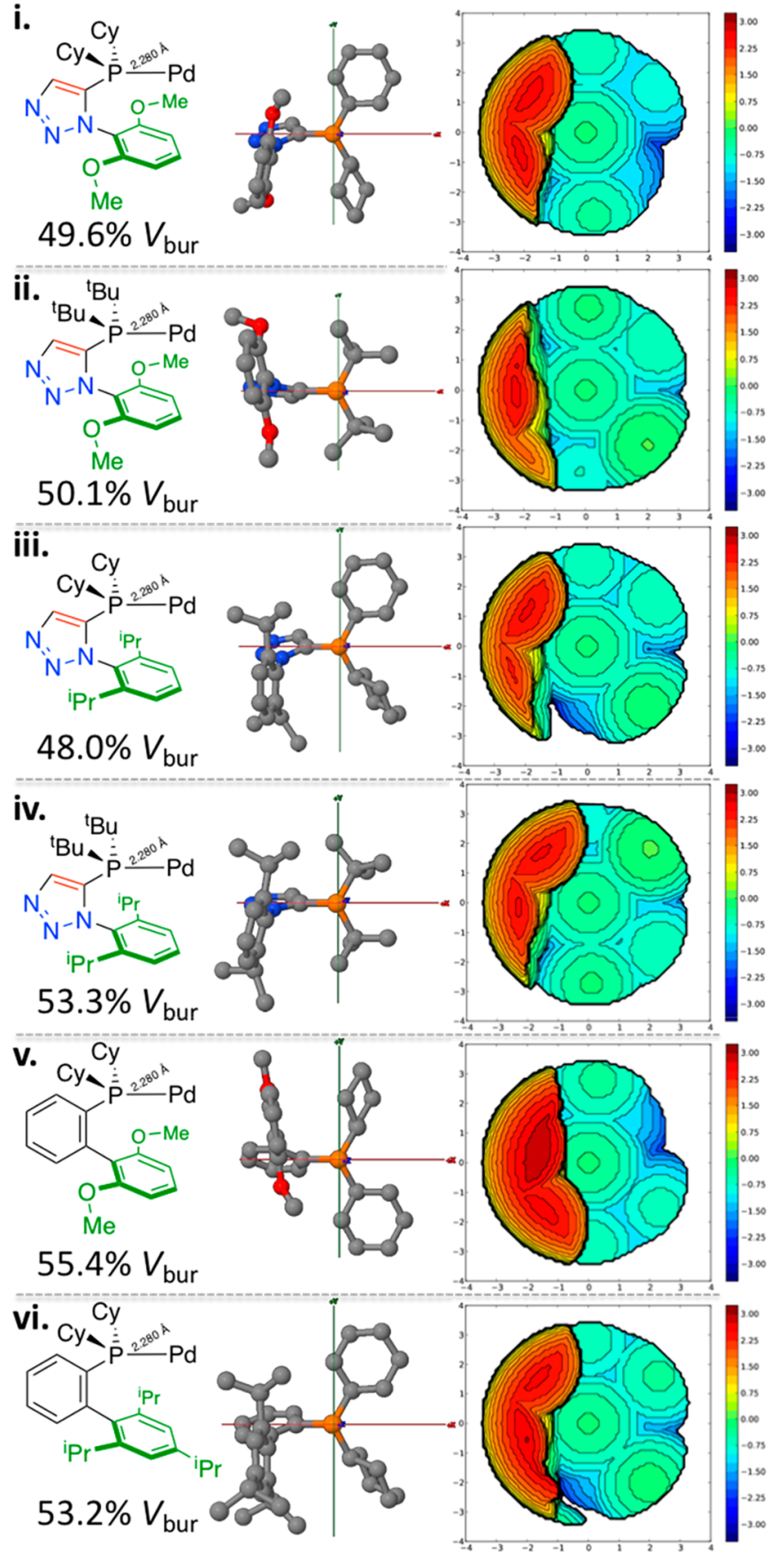

Figure 17. SambVca2.0 derived \% $V_{\text {bur }}$ and steric maps for the: (a) left side: (i) 29g; (ii) 29h; (iii) 29n; (iv) 29m; (v) 7 S-Phos; (vi) 8 X-Phos, derived palladium complexes of structures derived in silico and calculated with restricted dihedral angles as describe in Figure 15 (left); (b) right side: (i) 29g; (ii) 29h; (iii) 29n; (iv) 29m; (v) 7 S-Phos; (vi) 8 X-Phos, derived palladium complexes of structures derived in silico and calculated without any dihedral restrictions as describe in Figure 15 (right).

pounds). This illustrates the potential for this catalyst system to access both drug- and lead-like chemical space. Figure 10 (right) shows a PMI analysis of products $41 \mathbf{a}$ to $\mathbf{j}$, this analysis demonstrates a capability of this catalyst system to access nonflat Suzuki-Miyaura cross-coupled products.

These analyzes show that these compounds can be described as high-quality starting points for drug discovery programs.
These 10 compounds are now under evaluation for biological activity across a range of targets. ${ }^{125}$

Palladium Complexes. During the course of this study numerous attempts to grow crystals of palladium-phosphine complexes suitable for single crystal X-ray diffraction were made. Thus far, two attempts to generate X-ray quality crystals of palladium phosphine complexes have been achieved, using ligands $29 \mathrm{e}$ and $29 \mathrm{~h}$ with palladium(II) chloride. 
Table 6. Calculated (Spartan v16 and SambVca2.0) Steric Properties of 29e, 29g, 29h, 29m, 29n, S-Phos 7, and X-Phos 8

\begin{tabular}{|c|c|c|c|c|c|}
\hline entry & ligand & $\begin{array}{l}V_{\text {bur }} \text { calcd }[\%] \\
\text { (restricted) }\end{array}$ & $\begin{array}{l}V_{\text {bur }} \text { calcd }[\%] \\
\text { (unrestricted) }\end{array}$ & $\begin{array}{l}\text { Ar centroid-Pd distance }[\AA] \\
\text { (restricted) }\end{array}$ & $\begin{array}{l}\text { Ar centroid-Pd distance }[\AA] \\
\text { (unrestricted) }\end{array}$ \\
\hline 1 & $29 \mathrm{e}$ & $44.2(36.2)^{a}$ & 47.3 & $3.363(3.843(2))^{a}$ & 4.176 \\
\hline 2 & $29 g$ & $45.5(46.9)^{b}$ & 49.6 & $3.403(3.457)^{b}$ & 3.745 \\
\hline 3 & $29 \mathrm{~h}$ & 47.8 & 50.1 & 3.558 & 3.580 \\
\hline 4 & $29 \mathrm{~m}$ & 47.1 & 48.0 & 3.639 & 3.901 \\
\hline 5 & $29 n$ & 47.5 & 53.3 & 3.647 & 3.794 \\
\hline 6 & 7 (S-Phos) & $50.8(49.7)^{c}$ & 55.4 & 3.127 & 3.253 \\
\hline 7 & 8 (X-Phos) & $53.3(53.1)^{c}$ & 53.2 & 3.309 & 3.481 \\
\hline
\end{tabular}

${ }^{a}$ Measured from the unadjusted XRD structure of $42 .{ }^{b}$ Using free ligand $29 \mathrm{~g}$ XRD and applying Pd-P bond length $2.280 \AA$. . Refers to ligand/ metal 1:1 complex P-M distance $2.8 \mathrm{~A}(\mathrm{M}=\mathrm{Au}) .{ }^{12,129}$

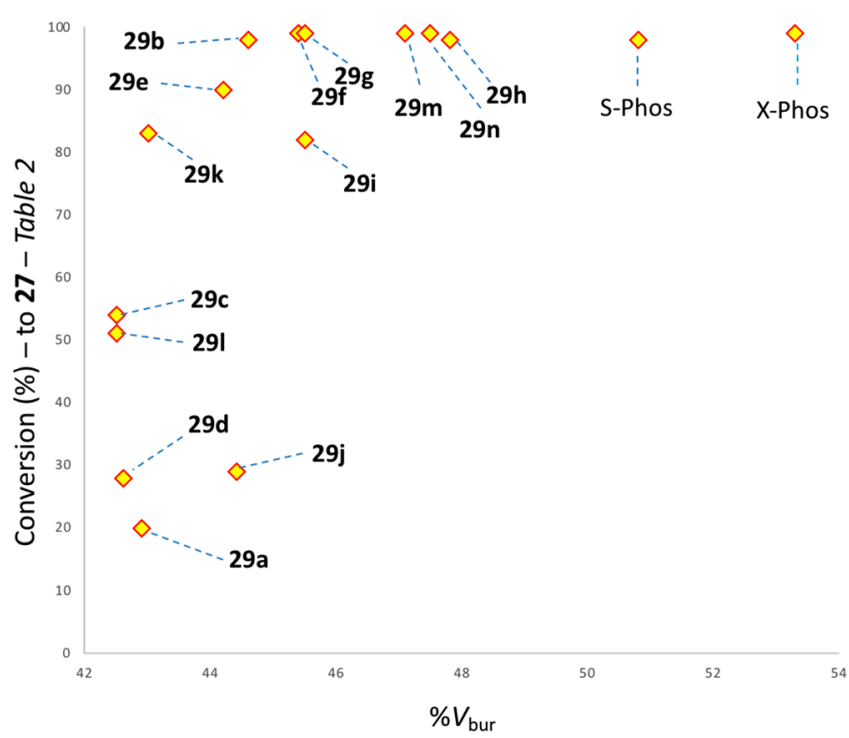

Figure 18. Conversion to $\mathbf{2 7}$ (see Table 2) versus computed burried volume (\%) as determined by the protocol discussed in Figure 15.

The combination of $29 \mathrm{e}$ and trans- $\mathrm{Pd}\left(\mathrm{CH}_{3} \mathrm{CN}\right)_{2} \mathrm{Cl}_{2}$ in dichloromethane at room temperature led to the formation of material that was precipitated by addition of pentane and the residue thus obtained was recrystallized from dichloromethane and hexane. A representation of the single crystal XRD structure of the palladium complex (42) thus obtained is depicted in Figure 11. A 2:1 ligand/metal square planar trans dichloride palladium(II) complex $\mathbf{4 2}$ was identified. Complex 42 may offer insight into the structural features of 29 series complexes as catalysts. The five-membered 1,5-disubstituted 1,2,3-triazole core presents the triazole 2,6-bismethoxy aryl fragment oriented toward the metal with an aryl-centroid $\cdots \mathrm{Pd}$ distance of $3.843(2) \AA$, in this solid-state structure. The triazole phosphine ligand offers a distinct geometric difference to phenylene core ligands (c.f., S-Phos and X-Phos, Figure 3), being more akin to other five-membered ring core ligands (c.f., 9 and 10, Figure 3), generating a slightly wider metal-binding pocket while still offering a stabilizing shield about the metal center.

The combination of $\mathbf{2 9 h}$ and trans- $\mathrm{Pd}\left(\mathrm{CH}_{3} \mathrm{CN}\right)_{2} \mathrm{Cl}_{2}$ in dichloromethane at room temperature led to the formation of material that was precepted by addition of pentane and recrystallized from dichloromethane and hexane. A representation of the single crystal XRD structure of the palladium complex (43) thus obtained is depicted in Figure 12. To our surprise, the XRD crystal structure shows $29 \mathrm{~h}$ functioning as a 3-N-coordinating ligand, with two such ligations are present about a trans-dichloride palladium(II) metal center. Since ligand $29 \mathrm{~h}$ functions as expected in the aforementioned catalyzed reactions, it is suggested that a more crystalline and readily formed (kinetic) complex is formed under the crystallization conditions employed. However, that this stable complex is formed reminds us of another potentially important feature of the triazole-core ligands, namely, a rear-side ancillary coordination point. It is conceivable that the ancillary nitrogen may offer some advantages in some catalyzed reaction, such as aggregation suppression for example. Suffice it to say, the sterically encumbered phosphine face of ligand $29 \mathrm{~h}$, as evidenced by the isolation of $\mathbf{4 3}$, bodes well for understanding differing catalysis modes of action on steric rationales as well as opportunities for divergence from phenylene core ligands.

Describing Phosphines. There is a growing body of literature discussing the importance of various parameters including steric effects ${ }^{116}$ of bulky phosphines ${ }^{83,84,126}$ relating to suitability and efficacy in catalysis (primarily as ligands for metals in metal mediated catalysis). ${ }^{108,115,127}$ While the Tolman cone angle has been an effective descriptor of ligand bulkiness for many years, ${ }^{111}$ it has been complimented more recently by Nolan's percentage buried volume parameter (\% $\left.V_{\text {bur }}\right){ }^{112}$ The $\% V_{\text {bur }}$ of ligands can be calculated using the SambVca (2.0) free web tool from Cavallo and co-workers; ${ }^{113,128}$ so we set about determining some steric parameters of our phosphines using this tool.

First, the crystal structure of the free ligand $29 \mathrm{~g}$ was investigated, as follows: The PDB file corresponding to the XRD crystal structure of ligand 29g was edited in Spartan'16 Parallel Suite (Wave function Inc.) by changing the valence of phosphorus to four and adding a palladium atom with a standard bond length of $2.280 \AA$ to generate a metalcoordinated model. The PBD file thus generated was not minimized or edited further, i.e., the atoms of the ligand remained in their crystallographically determined free-ligand position, and uploaded for analysis to the SambVca2.0 web tool for analysis. The added palladium atom was set as the center and then deleted; a summary of this analysis is shown in Figure 13. A steric map generated from this analysis is shown and a $47.0 \% V_{\text {bur }}$ was calculated, using otherwise default SambVca settings.

Following this analysis, the only palladium phosphine complex thus far successfully analyzed by single crystal X-ray diffraction studies (42) was investigated in a similar manner. Compound $\mathbf{4 2}$ is a square planar palladium(II) complex of ligand 29e and as such does not necessarily represent the catalytically relevant species, the determined $\% V_{\text {bur }}$ of $36.2 \%$ (Figure 14i) may not be the best comparator across a number of ligand structures, so three other forms of the complex were 
generated and compared. In order to find an appropriate comparison to fairly evaluate relative steric parameters of ligands $29 \mathrm{e}$ and $29 \mathrm{~g}$. The ligand portion of the crystal structure of 42 was edited in Spartan'16 Parallel Suite, the Pd-P bond length adjusted to $2.280 \AA$, and the SambVca-determined \% $V_{\text {bur }}$ of $36.9 \%$ (Figure 14ii) was essentially the same as that for the slightly longer, crystallographically determined, $\mathrm{Pd}-\mathrm{P}$ bond length in the earlier analysis. Since a model to allow for comparison of ligands where crystallographically determined data is not available was ultimately sought two further analyses were conducted.

Sigman and co-workers have previously used phosphine oxides as computational models for structural minimization proxies of ligand-metal complexes, ${ }^{115}$ so it was reasoned that computational minimization of in silico generated phosphine oxides may be a sensible starting point to allow for comparison among some of the ligands in this report. Two approaches were compared for optimizing and computationally determining the $\% V_{\text {bur }}$ of ligand 29e (summarized in Figure 15) using Spartan'16 Parallel Suite. In one case a phosphine oxide structure with no geometric restrictions was used (Figure 15, right); in the second case, the dihedral geometries of atoms 16 were restricted presenting the ligand's ancillary aryl group directly and orthogonally aligned to the coordination vector of the phosphine (the $\mathrm{P}-\mathrm{O}$ bond in this minimization), as shown in (Figure 15, left). Through comparison of the geometry unrestricted and geometry restricted protocols, with the structurally determined geometry (as shown in Figure 13) a protocol for analysis across the ligands of this report might be reasonably determined. In both cases the same minimization and optimization cascade was adopted; the structures used for computationally determined $\% V_{\text {bur }}$ calculations were obtained as follows. First, molecular mechanics (MMFF) conformer distribution (1000 max conformers) and subsequent molecular mechanics equilibrium geometries were ranked, and the 20 lowest energy conformers were retained for DFT investigation. The 20 retained conformers' equilibrium geometries were used as starting points for ground-state, gas phase equilibrium geometry determination (B3LYP 6-31G*). The lowest energy structure thus obtained was then edited to replace the oxygen (of the phosphine oxide) with a palladium atom and a standard P-Pd bond length of $2.280 \AA$ was applied (Figure 15, center).

The procedure outlined in Figure 15 was first applied to ligand 29e, and buried volumes of 44.2 and $47.3 \%$ were determined for the restricted and unrestricted geometries, respectively (Figure 16) and compared to the buried volume determined crystallographically. While this protocol gives slightly higher values, the restricted geometry model is more closely aligned to the XRD-derived model than the unrestricted one. With this information alone, it is difficult to ascertain if any one model provides a superior approach for assessing ligands where solid state data is not available. Furthermore, it should be remembered that the 42-derived \% $V_{\text {bur }}$ numbers are from solid-state structures and might not offer the best cross-ligand comparison. As such, the most active ligands of our study were examined using both protocols shown below (Figure 17).

Computed structures, percentage buried volumes, and steric maps of the six ligands of Figure 8, following the protocol outlined in Figure 15, are shown in Figure 17. The left side shows the information relating to restricted dihedral angle restricted complexes and the right side shows the structures obtained without imposing any geometrical restrictions (other than the $2.280 \AA \mathrm{P}-\mathrm{Pd}$ distances imposed throughout). It is interesting and confounded our expectations that the unrestricted geometry-minimized structures give a higher buried volume than the restricted dihedral angle structures (Figure 17 column a versus column b). Among the two data treatments, there is broad agreement with and relative similarity to each other. While the geometry-unrestricted structures of triazole ligands bear a striking resemblance (by rudimentary visual inspection) to any XRD-derived structures, the buried volume determinations of previously reported $S$ and $\mathrm{X}$-Phos (by the $\mathrm{P}=\mathrm{O}$ minimization proxy discussed earlier) complexes more closely match the geometry-restricted models we employed (Table 6, entries 6 and 7).

From the steric maps of Figure 17, it was noted that the space between the dark red bulky zone (to the left of the steric maps arising from the ancillary aryl groups of all ligands) protrudes in a manner to give more space between the central axis and red zone. Since space near the coordination site may be crucial in permitting reaction with bulky substrates, we also report the distance between the ancillary aryl group's centroid and the computed metal center (Table 6) for both the geometry-restricted and unrestricted ligands of Figure 8 are also listed in Table 6 (and contrasted against the data determined for ligand 29e). In Entry 2 the data arising from analysis of the previously detailed ligand crystal-structuredetermined structures of $\mathbf{2 9 g}$-complexes are given in parentheses. Between the restricted and unrestricted models of ligand-complex analysis, the centroid distance correlates more closely with the dihedral angle restricted model of a $29 \mathrm{~g}-\mathrm{Pd}$ complex.

It is notable that the $\mathrm{P}=\mathrm{O}$ ligand minimization protocol adopted gives good structural parameter agreement between those determined for palladium complexes of ligand $29 \mathrm{~g}$ both derived computationally and from a modified XRD structure of free ligand $29 \mathrm{~g}$ (Table 6, entry 1 ). By analyzing $\mathbf{2 9 a}-\mathbf{n}$ sideby-side using the same restricted dihedral angle minimization protocol (up to 10 conformers analyzed by DFT methods abbreviating the earlier minimization cascade for newly analyzed ligands) and plotting the computed $\% V_{\text {bur }}$ values against conversion to $\mathbf{2 7}$ (data from Table 2), as shown in Figure 18, it can be seen that a strong bulkiness versus conversion trend exists. Essentially any $\% V_{\text {bur }}$ value above $46 \%$ leads to quantitative conversion to products, under the prescribed conditions.

Further analysis of the data obtained for conversion in reactions catalyzed by 29 ligands is only against smaller data sets, and significant correlations of variances in conversions do not lead to any meaningfully comparable correlations. However, further study of cross-couplings of very bulky aryl chlorides may be warranted in the future since an intriguing balance between bulk and centroid distance is suggested (see the Supporting Information), but across only four data points surveyed to date, it may be too early to draw conclusion yet. ${ }^{130}$ A pre-peer-reviewed preprint of this article was deposited and may be viewed elsewhere. ${ }^{131}$

\section{CONCLUSIONS}

Two series of 1,2,3-triazole-containing 5-phosphino ligands were synthesized and tested as ligands in palladium catalyzed cross-coupling Suzuki-Miyaura reactions of bulky and heteroatom-containing substrates. The structural parameters of the 4-H triazole series (29) were determined by a restricted dihedral angle, phosphine oxide surrogate model, and a strong 
dependence upon bulkiness and catalytic activity were noted. Furthermore, a link to the space between the metal and the ancillary aryl group in the computed complexes was noted, suggesting bulkiness of ligand and space around the metal may both be implicated in delineating trends in cross-coupling of the most bulky and challenging substrates. Notably, triazolenitrogens' may not be completely innocent in the coordination environment created by these ligands, with a nitrogen coordination complex being characterized by XRD in one case. The ligands synthesized were benchmarked against commercially available ligands (X- and S-Phos) and in some cases the best triazole ligands match or outperformed them under the employed conditions, in like-for-like tests in triplicate. The phosphine ligands reported and characterized in this report represent easy to modify catalytic scaffolds that could be use in future library generation efforts and we are looking forward to facilitating access to these compounds and allowing others to include this type of ligand in their own catalyst screening campaigns.

\section{ASSOCIATED CONTENT}

\section{(S) Supporting Information}

The Supporting Information is available free of charge on the ACS Publications website at DOI: 10.1021/acs.organomet.8b00539.

Experimental procedures, spectral data, additional information, XRD collection parameters and CCDC deposit numbers (PDF)

Underpinning data of Figure 9 generated by LLAMA (PDF)

\section{Accession Codes}

CCDC 1856208-1856212 contain the supplementary crystallographic data for this paper. These data can be obtained free of charge via www.ccdc.cam.ac.uk/data_request/cif, or by emailingdata_request@ccdc.cam.ac.uk, or by contacting The Cambridge Crystallographic Data Centre, 12 Union Road, Cambridge CB2 1EZ, UK; fax: +44 1223336033.

\section{AUTHOR INFORMATION}

\section{Corresponding Author}

*E-mail: j.s.fossey@bham.ac.uk.

\section{ORCID}

Yiming Zhao: 0000-0003-2827-6511

Huy van Nguyen: 0000-0002-3985-1510

Louise Male: 0000-0002-8295-2528

Philip Craven: 0000-0002-7617-132X

Benjamin R. Buckley: 0000-0002-8028-0828

John S. Fossey: 0000-0002-2626-5117

\section{Author Contributions}

All authors contributed to the preparation of the manuscript, specific contributions in addition to this are listed for each coauthor in alphabetical order: B.R.B. helped direct aspects of the research, initiated some of the steric parameter analysis and gave input and critical assessment throughout the progress of the project; P.C. analyzed molecular property analysis through use of LLAMA and wrote aspects of the manuscript and ESI pertaining to this aspect; J.S.F. led and coconceived the project, providing critical assessment of data, day-to-day project management and oversight, directed all aspects throughout, supervised the experimental work and wrote the majority of the manuscript; L.M. is responsible for collecting, analyzing and preparing for publication single crystal XRD data, writing aspects of the main text and ESI and training oversight of Y.Z. in aspects of XRD data collection and analysis; H.v.N. conducted all the experiments of Scheme ${ }^{9}$, offered additional advice and wrote aspects of the ESI; Y.Z. coconceived aspects of the project, conducted all ligand synthesis and all-bar the Scheme ${ }^{9}$ reactions, wrote aspects of the main text, drafted a large proportion of the ESI, offered critical suggestions, under the mentorship of L.M. conducted some of the XRD data collection and analysis.

\section{Notes}

The authors declare no competing financial interest.

\section{ACKNOWLEDGMENTS}

J.S.F., Y.Z., P.C., H.v.N., and L.M. thanks the University of Birmingham for support. J.S.F. acknowledges the CASE consortium for networking opportunities. ${ }^{132,133}$ B.R.B. thanks Loughborough University and Research Councils UK for a RCUK Fellowship. J.S.F. thanks the Royal Society for an Industrial Fellowship and the EPSRC for funding (EP/ J003220/1). Dr. Chi Tsang and Dr. Peter Ashton are thanked for helpful discussions about mass spectrometry. Dr. Cécile $S$. Le Duff is thanked for advice on NMR spectroscopy. Helena Dodd is thanked for providing an initial data analysis and presentation app for MatLab. J.S.F. and B.R.B. acknowledge the support of a Wellcome Trust ISSF award within the University of Birmingham. J.S.F. and H.v.N. are thankful for the support of the NEURAM H2020 FET Open project 712821. All investigators are grateful for a Royal Society Research Grant $(2012 / \mathrm{R} 1)$ that underpins this project. The GSK free building block programme furnished a collection of boronic acids from which 40a was sourced. The University of Birmingham Scaffold Diversification Resource is gratefully acknowledged for providing access to a collection of compounds from which $39 a-f$ and $40 b-f$ were sourced.

\section{REFERENCES}

(1) Finn, M. G.; Kolb, H. C.; Fokin, V. V.; Sharpless, K. B. Click chemistry - Definition and aims. Prog. Chem. 2008, 20, 1-4.

(2) Kolb, H. C.; Finn, M. G.; Sharpless, K. B. Click chemistry: Diverse chemical function from a few good reactions. Angew. Chem., Int. Ed. 2001, 40, 2004.

(3) Lewis, W. G.; Green, L. G.; Grynszpan, F.; Radic, Z.; Carlier, P. R.; Taylor, P.; Finn, M. G.; Sharpless, K. B. Click chemistry in situ: Acetylcholinesterase as a reaction vessel for the selective assembly of a femtomolar inhibitor from an array of building blocks. Angew. Chem. Int. Ed. 2002, 41, 1053-1057.

(4) Rostovtsev, V. V.; Green, L. G.; Fokin, V. V.; Sharpless, K. B. A Stepwise Huisgen Cycloaddition Process: Copper(I)-Catalyzed Regioselective "Ligation" of Azides and Terminal Alkynes. Angew. Chem., Int. Ed. 2002, 41, 2596-2599.

(5) Tornøe, C. W.; Christensen, C.; Meldal, M. Peptidotriazoles on Solid Phase: [1,2,3]-Triazoles by Regiospecific Copper(I)-Catalyzed 1,3-Dipolar Cycloadditions of Terminal Alkynes to Azides. J. Org. Chem. 2002, 67, 3057-3064.

(6) Based upon citation reports using Thomas Reuters Web of Science TM for the term "click reaction".

(7) Meldal, M.; Tornøe, C. W. Cu-Catalyzed Azide-Alkyne Cycloaddition. Chem. Rev. 2008, 108, 2952-3015.

(8) Moses, J. E.; Moorhouse, A. D. The growing applications of click chemistry. Chem. Soc. Rev. 2007, 36, 1249-1262.

(9) Thirumurugan, P.; Matosiuk, D.; Jozwiak, K. Click Chemistry for Drug Development and Diverse Chemical-Biology Applications. Chem. Rev. 2013, 113, 4905-4979. 
(10) Amblard, F.; Cho, J. H.; Schinazi, R. F. Cu(I)-Catalyzed Huisgen Azide-Alkyne 1,3-Dipolar Cycloaddition Reaction in Nucleoside, Nucleotide, and Oligonucleotide Chemistry. Chem. Rev. 2009, 109, 4207-4220.

(11) El-Sagheer, A. H.; Brown, T. Click chemistry with DNA. Chem. Soc. Rev. 2010, 39, 1388-1405.

(12) Iha, R. K.; Wooley, K. L.; Nyström, A. M.; Burke, D. J.; Kade, M. J.; Hawker, C. J. Applications of Orthogonal "Click" Chemistries in the Synthesis of Functional Soft Materials. Chem. Rev. 2009, 109, $5620-5686$

(13) Schulze, B.; Schubert, U. S. Beyond click chemistry supramolecular interactions of 1,2,3-triazoles. Chem. Soc. Rev. 2014, $43,2522-2571$.

(14) Tron, G. C.; Pirali, T.; Billington, R. A.; Canonico, P. L.; Sorba, G.; Genazzani, A. A. Click chemistry reactions in medicinal chemistry: Applications of the 1,3-dipolar cycloaddition between azides and alkynes. Med. Res. Rev. 2008, 28, 278-308.

(15) Kolb, H. C.; Finn, M. G.; Sharpless, K. B. Click Chemistry: Diverse Chemical Function from a Few Good Reactions. Angew. Chem., Int. Ed. 2001, 40, 2004-2021.

(16) Zhang, Z.; Fan, E. Solid phase synthesis of peptidotriazoles with multiple cycles of triazole formation. Tetrahedron Lett. 2006, 47, 665669.

(17) Patterson, A. W.; Wood, W. J. L.; Hornsby, M.; Lesley, S.; Spraggon, G.; Ellman, J. A. Identification of Selective, Nonpeptidic Nitrile Inhibitors of Cathepsin S Using the Substrate Activity Screening Method. J. Med. Chem. 2006, 49, 6298-6307.

(18) Wood, W. J. L.; Patterson, A. W.; Tsuruoka, H.; Jain, R. K.; Ellman, J. A. Substrate Activity Screening: A Fragment-Based Method for the Rapid Identification of Nonpeptidic Protease Inhibitors. J. Am. Chem. Soc. 2005, 127, 15521-15527.

(19) Chuprakov, S.; Chernyak, N.; Dudnik, A. S.; Gevorgyan, V. Direct Pd-Catalyzed Arylation of 1,2,3-Triazoles. Org. Lett. 2007, 9, 2333-2336.

(20) Yamajala, K. D. B.; Patil, M.; Banerjee, S. Pd-Catalyzed Regioselective Arylation on the C-5 Position of N-Aryl 1,2,3Triazoles. J. Org. Chem. 2015, 80, 3003-3011.

(21) Winn, J.; Pinczewska, A.; Goldup, S. M. Synthesis of a Rotaxane $\mathrm{Cu}^{\mathrm{I}}$ Triazolide under Aqueous Conditions. J. Am. Chem. Soc. 2013, 135, 13318-13321.

(22) Ahlquist, M.; Fokin, V. V. Enhanced reactivity of dinuclear copper(I) acetylides in dipolar cycloadditions. Organometallics 2007, 26, 4389-4391.

(23) Buckley, B. R.; Dann, S. E.; Heaney, H. Experimental Evidence for the Involvement of Dinuclear Alkynylcopper(I) Complexes in Alkyne-Azide Chemistry. Chem. - Eur. J. 2010, 16, 6278-6284.

(24) Buckley, B. R.; Dann, S. E.; Harris, D. P.; Heaney, H.; Stubbs, E. C. Alkynylcopper(I) polymers and their use in a mechanistic study of alkyne-azide click reactions. Chem. Commun. 2010, 46, 2274-2276.

(25) Worrell, B. T.; Malik, J. A.; Fokin, V. V. Direct Evidence of a Dinuclear Copper Intermediate in $\mathrm{Cu}(\mathrm{I})$-Catalyzed Azide-Alkyne Cycloadditions. Science 2013, 340, 457-460.

(26) Makarem, A.; Berg, R.; Rominger, F.; Straub, B. F. A Fluxional Copper Acetylide Cluster in CuAAC Catalysis. Angew. Chem., Int. Ed. 2015, 54, 7431-7435.

(27) Elliott, P. I. P. Organometallic complexes with 1,2,3-triazolederived ligands. In Organometallic Chemistry; Fairlamb, I. J. S., Lynam, J. M., Eds.; The Royal Society of Chemistry, 2014; Vol. 39, Chapter 1, pp $1-25$.

(28) Schweinfurth, D.; Pattacini, R.; Strobel, S.; Sarkar, B. New 1,2,3-triazole ligands through click reactions and their palladium and platinum complexes. Dalton Trans 2009, 9291-9297.

(29) Kumar, S.; Saleem, F.; Singh, A. K. 'Click' generated 1,2,3triazole based organosulfur/selenium ligands and their $\mathrm{Pd}(\mathrm{II})$ and $\mathrm{Ru}$ (II) complexes: their synthesis, structure and catalytic applications. Dalton Trans 2016, 45, 11445-11458.

(30) Zhang, G.; Wang, Y.; Wen, X.; Ding, C.; Li, Y. Dual-functional click-triazole: a metal chelator and immobilization linker for the construction of a heterogeneous palladium catalyst and its application for the aerobic oxidation of alcohols. Chem. Commun. 2012, 48, 2979-2981.

(31) Hohloch, S.; Schweinfurth, D.; Sommer, M. G.; Weisser, F.; Deibel, N.; Ehret, F.; Sarkar, B. The redox series $\left[\mathrm{Ru}(\text { bpy })_{2}(\mathrm{~L})\right]^{n}, \mathrm{n}=$ $+3,+2,+1,0$, with $L=$ bipyridine, "click" derived pyridyl-triazole or bis-triazole: a combined structural, electrochemical, spectroelectrochemical and DFT investigation. Dalton Trans 2014, 43, 4437-4450.

(32) McCarney, E. P.; Hawes, C. S.; Blasco, S.; Gunnlaugsson, T. Synthesis and structural studies of 1,4-di(2-pyridyl)-1,2,3-triazole dpt and its transition metal complexes; a versatile and subtly unsymmetric ligand. Dalton Trans 2016, 45, 10209-10221.

(33) Bratsos, I.; Urankar, D.; Zangrando, E.; Genova-Kalou, P.; Kosmrlj, J.; Alessio, E.; Turel, I. 1-(2-Picolyl)-substituted 1,2,3triazole as novel chelating ligand for the preparation of ruthenium complexes with potential anticancer activity. Dalton Trans 2011, 40, 5188-5199.

(34) Chowdhury, B.; Khatua, S.; Dutta, R.; Chakraborty, S.; Ghosh, P. Bis-Heteroleptic Ruthenium(II) Complex of a Triazole Ligand as a Selective Probe for Phosphates. Inorg. Chem. 2014, 53, 8061-8070.

(35) Fernández-Hernández, J. M.; Yang, C.-H.; Beltrán, J. I.; Lemaur, V.; Polo, F.; Fröhlich, R.; Cornil, J.; De Cola, L. Control of the Mutual Arrangement of Cyclometalated Ligands in Cationic Iridium(III) Complexes. Synthesis, Spectroscopy, and Electroluminescence of the Different Isomers. J. Am. Chem. Soc. 2011, 133, 10543-10558.

(36) Swanick, K. N.; Ladouceur, S.; Zysman-Colman, E.; Ding, Z. Bright electrochemiluminescence of iridium(III) complexes. Chem. Commun. 2012, 48, 3179-3181.

(37) Ladouceur, S.; Fortin, D.; Zysman-Colman, E. Enhanced Luminescent Iridium(III) Complexes Bearing Aryltriazole Cyclometallated Ligands. Inorg. Chem. 2011, 50, 11514-11526.

(38) Chan, T. R.; Hilgraf, R.; Sharpless, K. B.; Fokin, V. V. Polytriazoles as Copper(I)-Stabilizing Ligands in Catalysis. Org. Lett. 2004, 6, 2853-2855.

(39) Michaels, H. A.; Zhu, L. Ligand-Assisted, Copper(II) AcetateAccelerated Azide-Alkyne Cycloaddition. Chem. - Asian J. 2011, 6, $2825-2834$.

(40) Mathew, P.; Neels, A.; Albrecht, M. 1,2,3-Triazolylidenes as Versatile Abnormal Carbene Ligands for Late Transition Metals. J. Am. Chem. Soc. 2008, 130, 13534-13535.

(41) Kilpin, K. J.; Paul, U. S. D.; Lee, A.-L.; Crowley, J. D. Gold(i) "click" 1,2,3-triazolylidenes: synthesis, self-assembly and catalysis. Chem. Commun. 2011, 47, 328-330.

(42) Crowley, J. D.; Lee, A.-L.; Kilpin, K. J. 1,3,4-Trisubstituted1,2,3-Triazol-5-ylidene 'Click' Carbene Ligands: Synthesis, Catalysis and Self-Assembly. Aust. J. Chem. 2011, 64, 1118-1132.

(43) Wright, J. R.; Young, P. C.; Lucas, N. T.; Lee, A.-L.; Crowley, J. D. Gold(I) and Palladium(II) Complexes of 1,3,4-Trisubstituted 1,2,3-Triazol-5-ylidene "Click" Carbenes: Systematic Study of the Electronic and Steric Influence on Catalytic Activity. Organometallics 2013, 32, 7065-7076.

(44) Bouffard, J.; Keitz, B. K.; Tonner, R.; Guisado-Barrios, G.; Frenking, G.; Grubbs, R. H.; Bertrand, G. Synthesis of Highly Stable 1,3-Diaryl-1H-1,2,3-triazol-5-ylidenes and Their Applications in Ruthenium-Catalyzed Olefin Metathesis. Organometallics 2011, 30, 2617-2627.

(45) Nakamura, T.; Terashima, T.; Ogata, K.; Fukuzawa, S.-i. Copper(I) 1,2,3-Triazol-5-ylidene Complexes as Efficient Catalysts for Click Reactions of Azides with Alkynes. Org. Lett. 2011, 13, 620623.

(46) Flores-Jarillo, M.; Mendoza-Espinosa, D.; Salazar-Pereda, V.; González-Montiel, S. Synthesis and Catalytic Benefits of Tetranuclear Gold(I) Complexes with a $C_{4}$-Symmetric Tetratriazol-5-ylidene. Organometallics 2017, 36, 4305-4312.

(47) Schmid, J.; Frey, W.; Peters, R. Polynuclear Enantiopure Salen-Mesoionic Carbene Hybrid Complexes. Organometallics 2017, 36, 4313-4324.

(48) Kinzhalov, M. A.; Legkodukh, A. S.; Anisimova, T. B.; Novikov, A. S.; Suslonov, V. V.; Luzyanin, K. V.; Kukushkin, V. Y. Tetrazol-5- 
ylidene Gold(III) Complexes from Sequential [2 + 3] Cycloaddition of Azide to Metal-Bound Isocyanides and N4 Alkylation. Organometallics 2017, 36, 3974-3980.

(49) Schaper, L.-A.; Wei, X.; Hock, S. J.; Pöthig, A.; Öfele, K.; Cokoja, M.; Herrmann, W. A.; Kühn, F. E. Gold(I) Complexes with "Normal" 1,2,3-Triazolylidene Ligands: Synthesis and Catalytic Properties. Organometallics 2013, 32, 3376-3384.

(50) Huang, D.; Zhao, P.; Astruc, D. Catalysis by 1,2,3-triazole- and related transition-metal complexes. Coord. Chem. Rev. 2014, 272, $145-165$.

(51) Scrafton, D. K.; Taylor, J. E.; Mahon, M. F.; Fossey, J. S.; James, T. D. Click-fluors": Modular fluorescent saccharide sensors based on a 1,2,3-triazole ring. J. Org. Chem. 2008, 73, 2871-2874.

(52) Zhai, W.; Chapin, B. M.; Yoshizawa, A.; Wang, H.-C.; Hodge, S. A.; James, T. D.; Anslyn, E. V.; Fossey, J. S. Click-fluors": triazolelinked saccharide sensors. Org. Chem. Front. 2016, 3, 918-928.

(53) Zhai, W.; Male, L.; Fossey, J. S. Glucose selective bis-boronic acid click-fluor. Chem. Commun. 2017, 53, 2218-2221.

(54) Brittain, W. D.; Buckley, B.; Fossey, J. S. Asymmetric "click" chemistry focusing on the copper catalyzed azide-alkyne cycloaddition. Abstr. Pap. - Am. Chem. Soc., 2015, CATL-207.

(55) Brittain, W. D. G.; Buckley, B. R.; Fossey, J. S. Kinetic resolution of alkyne-substituted quaternary oxindoles via copper catalysed azide-alkyne cycloadditions. Chem. Commun. 2015, 51, 17217-17220.

(56) Brittain, W. D. G.; Chapin, B. M.; Zhai, W.; Lynch, V. M.; Buckley, B. R.; Anslyn, E. V.; Fossey, J. S. The Bull-James assembly as a chiral auxiliary and shift reagent in kinetic resolution of alkyne amines by the CuAAC reaction. Org. Biomol. Chem. 2016, 14, 1077810782 .

(57) Brittain, W. D. G.; Dalling, A. G.; Sun, Z.; Le Duff, C. S.; Male, L.; Buckley, B. R.; Fossey, J. S. Simultaneous Clicknetic Resolution Coetaneous catalytic kinetic resolution of alkynes and azides through asymmetric triazole formation. ChemRxiv 2017, DOI: 10.26434/ chemrxiv.5663623.v1.

(58) Brittain, W. D. G.; Buckley, B. R.; Fossey, J. S. Asymmetric Copper-Catalyzed Azide-Alkyne Cycloadditions. ACS Catal. 2016, 6, $3629-3636$

(59) Fossey, J. S.; James, T. D. Boronic Acid Based Modular Fluorescent Saccharide Sensors. Rev. Fluoresc. 2009, 2007, 103-118.

(60) Fossey, J. S.; James, T. D. Boronic acid based receptors. Supramol. Chem.: Mol. Nanomater. 2012, 2, 1346-1379.

(61) Nishiyabu, R.; Kubo, Y.; James, T. D.; Fossey, J. S. Boronic acid building blocks: tools for sensing and separation. Chem. Commun. 2011, 47, 1106-1123.

(62) Nishiyabu, R.; Kubo, Y.; James, T. D.; Fossey, J. S. Boronic acid building blocks: tools for self assembly. Chem. Commun. 2011, 47, $1124-1150$.

(63) Bull, S. D.; Davidson, M. G.; van den Elsen, J. M. H.; Fossey, J. S.; Jenkins, A. T. A.; Jiang, Y.-B.; Kubo, Y.; Marken, F.; Sakurai, K.; Zhao, J.; James, T. D. Exploiting the Reversible Covalent Bonding of Boronic Acids: Recognition, Sensing, and Assembly. Acc. Chem. Res. 2013, 46, 312-326.

(64) Zhai, W.; Sun, X.; James, T. D.; Fossey, J. S. Boronic AcidBased Carbohydrate Sensing. Chem. - Asian J. 2015, 10, 1836-1848.

(65) Xu, S. Y.; Wang, H. C.; Flower, S. E.; Fossey, J. S.; Jiang, Y. B.; James, T. D. Suzuki homo-coupling reaction based fluorescent sensors for monosaccharides. RSC Adv. 2014, 4, 35238-35241.

(66) Buckley, B. R. Recent Advances/Contributions in the SuzukiMiyaura Reaction. Boron: Sensing, Synthesis and Supramolecular SelfAssembly 2015, 389-409.

(67) Johansson Seechurn, C. C. C.; Kitching, M. O.; Colacot, T. J.; Snieckus, V. Palladium-Catalyzed Cross-Coupling: A Historical Contextual Perspective to the 2010 Nobel Prize. Angew. Chem., Int. Ed. 2012, 51, 5062-5085.

(68) Littke, A. F.; Fu, G. C. Palladium-catalyzed coupling reactions of aryl chlorides. Angew. Chem., Int. Ed. 2002, 41, 4176-4211.

(69) Phan, N. T. S.; Van Der Sluys, M.; Jones, C. W. On the Nature of the Active Species in Palladium Catalyzed Mizoroki-Heck and
Suzuki-Miyaura Couplings - Homogeneous or Heterogeneous Catalysis, A Critical Review. Adv. Synth. Catal. 2006, 348, 609-679.

(70) Buchwald, S. L. Transition metal-catalyzed cross-coupling and the Heck coupling processes: Powerful reactions for carbon-carbon and carbon-heteroatom bond formation. Adv. Synth. Catal. 2004, 346, $1524-1524$

(71) Miyaura, N. Heck and cross-coupling reactions: Two core chemistries in metal-catalyzed organic syntheses. Adv. Synth. Catal. 2004, 346, 1522-1523.

(72) Martin, R.; Buchwald, S. L. Palladium-Catalyzed SuzukiMiyaura Cross-Coupling Reactions Employing Dialkylbiaryl Phosphine Ligands. Acc. Chem. Res. 2008, 41, 1461-1473.

(73) Ishikawa, S.; Manabe, K. Synthetic method for multifunctionalized oligoarenes using pinacol esters of hydroxyphenylboronic acids. Chem. Commun. 2006, 2589-2591.

(74) Rygus, J. P. G.; Crudden, C. M. Enantiospecific and Iterative Suzuki-Miyaura Cross-Couplings. J. Am. Chem. Soc. 2017, 139, 18124-18137.

(75) Kozuch, S.; Martin, J. M. L. What Makes for a Bad Catalytic Cycle? A Theoretical Study on the Suzuki-Miyaura Reaction within the Energetic Span Model. ACS Catal. 2011, 1, 246-253.

(76) Zhou, Y.; Zhang, X.; Liang, H.; Cao, Z.; Zhao, X.; He, Y.; Wang, S.; Pang, J.; Zhou, Z.; Ke, Z.; Qiu, L. Enantioselective Synthesis of Axially Chiral Biaryl Monophosphine Oxides via Direct Asymmetric Suzuki Coupling and DFT Investigations of the Enantioselectivity. ACS Catal. 2014, 4, 1390-1397.

(77) Kutchukian, P. S.; Dropinski, J. F.; Dykstra, K. D.; Li, B.; DiRocco, D. A.; Streckfuss, E. C.; Campeau, L.-C.; Cernak, T.; Vachal, P.; Davies, I. W.; Krska, S. W.; Dreher, S. D. Chemistry informer libraries: a chemoinformatics enabled approach to evaluate and advance synthetic methods. Chem. Sci. 2016, 7, 2604-2613.

(78) Littke, A. F.; Dai, C. Y.; Fu, G. C. Versatile catalysts for the Suzuki cross-coupling of arylboronic acids with aryl and vinyl halides and triflates under mild conditions. J. Am. Chem. Soc. 2000, 122, $4020-4028$.

(79) Hundertmark, T.; Littke, A. F.; Buchwald, S. L.; Fu, G. C. $\mathrm{Pd}(\mathrm{PhCN})_{2} \mathrm{Cl}_{2} / \mathrm{P}(t-\mathrm{Bu})_{3}$ : A versatile catalyst for sonogashira reactions of aryl bromides at room temperature. Org. Lett. 2000, 2, $1729-1731$

(80) Littke, A. F.; Fu, G. C. Heck reactions in the presence of $\mathrm{P}(t$ $\mathrm{Bu})_{3}$ : Expanded scope and milder reaction conditions for the coupling of aryl chlorides. J. Org. Chem. 1999, 64, 10-11.

(81) Littke, A. F.; Fu, G. C. A convenient and general method for Pd-catalyzed Suzuki cross-couplings of aryl chlorides and arylboronic acids. Angew. Chem., Int. Ed. 1998, 37, 3387-3388.

(82) Fu, G. C. The Development of Versatile Methods for Palladium-Catalyzed Coupling Reactions of Aryl Electrophiles through the Use of $\mathrm{P}(t-\mathrm{Bu})_{3}$ and $\mathrm{PCy}_{3}$ as Ligands. Acc. Chem. Res. 2008, 41, 1555-1564.

(83) Su, M.; Buchwald, S. L. A Bulky Biaryl Phosphine Ligand Allows for Palladium-Catalyzed Amidation of Five-Membered Heterocycles as Electrophiles. Angew. Chem., Int. Ed. 2012, 51, $4710-4713$.

(84) Altman, R. A.; Buchwald, S. L. Pd-catalyzed Suzuki-Miyaura reactions of aryl halides using bulky biarylmonophosphine ligands. Nat. Protoc. 2007, 2, 3115-3121.

(85) Barder, T. E.; Walker, S. D.; Martinelli, J. R.; Buchwald, S. L. Catalysts for Suzuki-Miyaura Coupling Processes: Scope and Studies of the Effect of Ligand Structure. J. Am. Chem. Soc. 2005, 127, 46854696.

(86) Huang, X.; Anderson, K. W.; Zim, D.; Jiang, L.; Klapars, A.; Buchwald, S. L. Expanding Pd-Catalyzed C-N Bond-Forming Processes: The First Amidation of Aryl Sulfonates, Aqueous Amination, and Complementarity with Cu-Catalyzed Reactions. J. Am. Chem. Soc. 2003, 125, 6653-6655.

(87) Bruno, N. C.; Tudge, M. T.; Buchwald, S. L. Design and preparation of new palladium precatalysts for C-C and C-N crosscoupling reactions. Chem. Sci. 2013, 4, 916-920. 
(88) Bruno, N. C.; Niljianskul, N.; Buchwald, S. L. N-Substituted 2Aminobiphenylpalladium Methanesulfonate Precatalysts and Their Use in $\mathrm{C}-\mathrm{C}$ and $\mathrm{C}-\mathrm{N}$ Cross-Couplings. J. Org. Chem. 2014, 79, 4161-4166.

(89) Bedford, R. B.; Cazin, C. S. J.; Holder, D. The development of palladium catalysts for $\mathrm{C}-\mathrm{C}$ and $\mathrm{C}$-heteroatom bond forming reactions of aryl chloride substrates. Coord. Chem. Rev. 2004, 248, 2283-2321.

(90) Zapf, A.; Jackstell, R.; Rataboul, F.; Riermeier, T.; Monsees, A.; Fuhrmann, C.; Shaikh, N.; Dingerdissen, U.; Beller, M. Practical synthesis of new and highly efficient ligands for the Suzuki reaction of aryl chlorides. Chem. Commun. 2004, 38-39.

(91) Zapf, A.; Sundermeier, M.; Jackstell, R.; Beller, M.; Riermeier, T.; Monsees, A.; Dingerdissen, U. Nitrogen-containing monodentate phosphines and their use in catalysis. WO2004101581A2, 2004.

(92) Pickett, T. E.; Roca, F. X.; Richards, C. J. Synthesis of Monodentate Ferrocenylphosphines and Their Application to the Palladium-Catalyzed Suzuki Reaction of Aryl Chlorides. J. Org. Chem. 2003, 68, 2592-2599.

(93) Jensen, J. F.; Johannsen, M. New Air-Stable Planar Chiral Ferrocenyl Monophosphine Ligands: Suzuki Cross-Coupling of Aryl Chlorides and Bromides. Org. Lett. 2003, 5, 3025-3028.

(94) Liu, S.-Y.; Choi, M. J.; Fu, G. C. A surprisingly mild and versatile method for palladium-catalyzed Suzuki cross-couplings of aryl chlorides in the presence of a triarylphosphine. Chem. Commun. 2001, 2408-2409.

(95) Dubrovina, N. V.; Domke, L.; Shuklov, I. A.; Spannenberg, A.; Franke, R.; Villinger, A.; Börner, A. New mono- and bidentate Pligands using one-pot click-chemistry: synthesis and application in Rhcatalyzed hydroformylation. Tetrahedron 2013, 69, 8809-8817.

(96) Schuster, E. M.; Nisnevich, G.; Botoshansky, M.; Gandelman, M. Synthesis of Novel Bulky, Electron-Rich Propargyl and Azidomethyl Dialkyl Phosphines and Their Use in the Preparation of Pincer Click Ligands. Organometallics 2009, 28, 5025-5031.

(97) Dolhem, F.; Johansson, M. J.; Antonsson, T.; Kann, N. Modular Synthesis of ChiraClick Ligands: A Library of P-Chirogenic Phosphines. J. Comb. Chem. 2007, 9, 477-486.

(98) Liu, D.; Gao, W.; Dai, Q.; Zhang, X. Triazole-Based Monophosphines for Suzuki-Miyaura Coupling and Amination Reactions of Aryl Chlorides. Org. Lett. 2005, 7, 4907-4910.

(99) Dai, Q.; Gao, W.; Liu, D.; Kapes, L. M.; Zhang, X. TriazoleBased Monophosphine Ligands for Palladium-Catalyzed CrossCoupling Reactions of Aryl Chlorides. J. Org. Chem. 2006, 71, 3928-3934.

(100) Zhang, X. Clickphosphines for transition metal-catalyzed reactions. WO2006130842A1, 2006.

(101) Zink, D. M.; Baumann, T.; Nieger, M.; Bräse, S. Improved One-Pot Synthesis of $\mathrm{C}_{3}$-Symmetric ClickPhos and Related Ligands: Structures of Unique Triazole-Zinc Complexes. Eur. J. Org. Chem. 2011, 2011, 1432-1437.

(102) Choubey, B.; Radhakrishna, L.; Mague, J. T.; Balakrishna, M. S. Two Triazole-Based Phosphine Ligands Prepared via TemperatureMediated $\mathrm{Li} / \mathrm{H}$ Exchange: $\mathrm{CuI}$ and AuI Complexes and Structural Studies. Inorg. Chem. 2016, 55, 8514-8526.

(103) Oki, H.; Oura, I.; Nakamura, T.; Ogata, K.; Fukuzawa, S.-i. Modular synthesis of the ClickFerrophos ligand family and their use in rhodium- and ruthenium-catalyzed asymmetric hydrogenation. Tetrahedron: Asymmetry 2009, 20, 2185-2191.

(104) Glover, J. E.; Martin, D. J.; Plieger, P. G.; Rowlands, G. J. Planar Chiral Triazole-Based Phosphanes Derived from [2.2]Paracyclophane and Their Activity in Suzuki Coupling Reactions. Eur. J. Org. Chem. 2013, 2013, 1671-1675.

(105) Austeri, M.; Enders, M.; Nieger, M.; Bräse, S. [2.2]Paracyclophane-Triazolyl Monophosphane Ligands: Synthesis and Their Copper and Palladium Complexes. Eur. J. Org. Chem. 2013, 2013, 1667-1670.

(106) Laborde, C.; Wei, M.-M.; van der Lee, A.; Deydier, E.; Daran, J.-C.; Volle, J.-N.; Poli, R.; Pirat, J.-L.; Manoury, E.; Virieux, D. Double $[3+2]$-dimerisation cascade synthesis of bis(triazolyl)- bisphosphanes, a new scaffold for bidentate bisphosphanes. Dalton Trans 2015, 44, 12539-12545.

(107) Cao, L.; Huang, S.; Liu, W.; Yan, X. 4-Phosphino-1,2,3-triazol5-ylidenes: Stable Bridging Ligands for Coinage Metal Complexes. Organometallics 2018, 37, 2010-2013.

(108) Christian, A. H.; Niemeyer, Z. L.; Sigman, M. S.; Toste, F. D. Uncovering Subtle Ligand Effects of Phosphines Using Gold(I) Catalysis. ACS Catal. 2017, 7, 3973-3978.

(109) Barrett, M. J.; Khan, G. F.; Davies, P. W.; Grainger, R. S. Alkynyl sulfoxides as $\alpha$-sulfinyl carbene equivalents: gold-catalysed oxidative cyclopropanation. Chem. Commun. 2017, 53, 5733-5736.

(110) Santos, M. D.; Davies, P. W. A gold-catalysed fully intermolecular oxidation and sulfur-ylide formation sequence on ynamides. Chem. Commun. 2014, 50, 6001-6004.

(111) Tolman, C. A. Steric effects of phosphorus ligands in organometallic chemistry and homogeneous catalysis. Chem. Rev. 1977, 77, 313-348.

(112) Clavier, H.; Nolan, S. P. Percent buried volume for phosphine and N-heterocyclic carbene ligands: steric properties in organometallic chemistry. Chem. Commun. 2010, 46, 841-861.

(113) Poater, A.; Cosenza, B.; Correa, A.; Giudice, S.; Ragone, F.; Scarano, V.; Cavallo, L. SambVca: A Web Application for the Calculation of the Buried Volume of N-Heterocyclic Carbene Ligands. Eur. J. Inorg. Chem. 2009, 2009, 1759-1766.

(114) Wu, K.; Doyle, A. G. Parameterization of phosphine ligands demonstrates enhancement of nickel catalysis via remote steric effects. Nat. Chem. 2017, 9, 779-784.

(115) Niemeyer, Z. L.; Milo, A.; Hickey, D. P.; Sigman, M. S. Parameterization of phosphine ligands reveals mechanistic pathways and predicts reaction outcomes. Nat. Chem. 2016, 8, 610-617.

(116) Kendall, A. J.; Zakharov, L. N.; Tyler, D. R. Steric and Electronic Influences of Buchwald-Type Alkyl-JohnPhos Ligands. Inorg. Chem. 2016, 55, 3079-3090.

(117) Lovering, F.; Bikker, J.; Humblet, C. Escape from Flatland: Increasing Saturation as an Approach to Improving Clinical Success. J. Med. Chem. 2009, 52, 6752-6756.

(118) Colomer, I.; Empson, C. J.; Craven, P.; Owen, Z.; Doveston, R. G.; Churcher, I.; Marsden, S. P.; Nelson, A. A divergent synthetic approach to diverse molecular scaffolds: assessment of lead-likeness using LLAMA, an open-access computational tool. Chem. Commun. 2016, 52, 7209-7212.

(119) Arthurs, R. A.; Richards, C. J. Deuterium as a Stereochemically Invisible Blocking Group for Chiral Ligand Synthesis. Org. Lett. 2017, 19, 702-705.

(120) Adamo, C.; Amatore, C.; Ciofini, I.; Jutand, A.; Lakmini, H. Mechanism of the Palladium-Catalyzed Homocoupling of Arylboronic Acids: Key Involvement of a Palladium Peroxo Complex. J. Am. Chem. Soc. 2006, 128, 6829-6836.

(121) Vitaku, E.; Smith, D. T.; Njardarson, J. T. Analysis of the Structural Diversity, Substitution Patterns, and Frequency of Nitrogen Heterocycles among U.S. FDA Approved Pharmaceuticals. J. Med. Chem. 2014, 57, 10257-10274.

(122) Taylor, R. D.; MacCoss, M.; Lawson, A. D. G. Rings in Drugs. J. Med. Chem. 2014, 57, 5845-5859.

(123) Wang, J.; Hou, T. Drug and Drug Candidate Building Block Analysis. J. Chem. Inf. Model. 2010, 50, 55-67.

(124) The University of Birmingham Scaffold Diversification Resource is a collection of reagents designed to contain functionality commonly found in medicinal chemistry, to allow the late-stage diversification of molecular scaffolds.

(125) Should biological activity be observed, and the results deemed of consequence, subsequent future study and resulting reports will be directed towards exploring scope and SAR.

(126) Chen, L.; Ren, P.; Carrow, B. P. Tri(1-adamantyl)phosphine: Expanding the Boundary of Electron-Releasing Character Available to Organophosphorus Compounds. J. Am. Chem. Soc. 2016, 138, 63926395. 
(127) Wu, K.; Doyle, A. G. Parameterization of phosphine ligands demonstrates enhancement of nickel catalysis via remote steric effects. Nat. Chem. 2017, 9, 779.

(128) Falivene, L.; Credendino, R.; Poater, A.; Petta, A.; Serra, L.; Oliva, R.; Scarano, V.; Cavallo, L. SambVca 2. A Web Tool for Analyzing Catalytic Pockets with Topographic Steric Maps. Organometallics 2016, 35, 2286-2293.

(129) Partyka, D. V.; Robilotto, T. J.; Zeller, M.; Hunter, A. D.; Gray, T. G. Dialkylbiarylphosphine complexes of gold(I) halides. Gold-aryl $\pi$-interactions in the solid state. Organometallics 2008, 27, 28-32.

(130) At this stage, structural parameters for ligand series $\mathbf{1 8}$ have not been determined to a level of satisfactory confidence to permit inclusion in this report. Without XRD data to corroborate any assumptions made in structural minimisations too many uncertainties remain in drawing any conclusions from imposed correlations.

(131) Zhao, Y.; Van Nguyen, H.; Male, L.; Craven, P.; Buckley, B. R.; Fossey, J. S. Phosphino-Triazole Ligands for Palladium-Catalysed Cross-Coupling. ChemRxiv 2018, DOI: 10.26434/chemrxiv.6823259.v1.

(132) Fossey, J. S.; Brittain, W. D. G. The CASE 2014 symposium: Catalysis and sensing for our environment, Xiamen 7th-9th November 2014. Org. Chem. Front. 2015, 2, 101-105.

(133) Payne, D. T.; Fossey, J. S.; Elmes, R. B. P. Catalysis and Sensing for our Environment (CASE2015) and the Supramolecular Chemistry Ireland Meeting (SCI 2015): Dublin and Maynooth, Ireland. 8th-11th July. Supramol. Chem. 2016, 28, 921-931. 